\title{
A microscopic description of absorption in high-energy string-brane collisions
}

\author{
Giuseppe D’Appollonio, ${ }^{a}$ Paolo Di Vecchia, ${ }^{b, c}$ Rodolfo Russo ${ }^{d}$ \\ and Gabriele Veneziano ${ }^{e, f}$ \\ ${ }^{a}$ Dipartimento di Fisica, Università di Cagliari and INFN \\ Cittadella Universitaria, 09042 Monserrato, Italy \\ ${ }^{b}$ The Niels Bohr Institute, University of Copenhagen, \\ Blegdamsvej 17, DK-2100 Copenhagen Ø, Denmark \\ ${ }^{c}$ Nordita, KTH Royal Institute of Technology and Stockholm University, \\ Roslagstullsbacken 23, SE-10691 Stockholm, Sweden \\ ${ }^{d}$ Queen Mary University of London, \\ Mile End Road, E1 4NS London, United Kingdom \\ ${ }^{e}$ Collège de France, \\ 11 place M. Berthelot, 75005 Paris, France \\ f Theory Division, CERN, \\ CH-1211 Geneva 23, Switzerland \\ E-mail: giuseppe.dappollonio@ca.infn.it, divecchi@nbi.dk, \\ r.russo@qmul.ac.uk, gabriele.veneziano@cern.ch
}

ABSTRACT: We study the collision of a highly energetic light closed string off a stack of $\mathrm{D} p$-branes at (sub)string-scale impact parameters and in a regime justifying a perturbative treatment. Unlike at larger impact parameters - where elastic scattering and/or tidal excitations dominate - here absorption of the closed string by the brane system, with the associated excitation of open strings living on it, becomes important. As a first step, we study this phenomenon at the disk level, in which the energetic closed string turns into a single heavy open string at rest whose particularly simple properties are described.

KeYwords: D-branes, Bosonic Strings

ARXIV EPRINT: 1510.03837 


\section{Contents}

1 Introduction 1

2 Elastic scattering of closed strings by D-branes 4

3 Absorption of closed strings by D-branes 10

$\begin{array}{lll}3.1 & \text { Closed-open amplitudes } & 11\end{array}$

$\begin{array}{lll}3.2 & \text { The closed-open string vertex } & 12\end{array}$

4 The high-energy limit of the closed-open vertex 16

5 Closed-open transitions at high energy 18

$\begin{array}{ll}5.1 \text { Tachyon } & 19\end{array}$

5.2 Massless states 24

$\begin{array}{lll}5.3 & \text { First massive level } & 26\end{array}$

5.4 Generic massive states 31

6 Conclusions 33

A The light-cone vertex and the covariant amplitudes $\quad 34$

A.1 Tachyon to leading Regge 34

A.2 Massless to leading Regge 36

B On the calculation of $\operatorname{Im} \mathcal{A}$ from the vertex 39

\section{Introduction}

The dynamics of strings in the background of a collection of $\mathrm{D} p$-branes provides an excellent framework to address the problem of string dynamics in curved spacetimes. It also underlies many important developments in our understanding of quantum gravity, most notably the gauge-gravity duality [1-3].

In a series of relatively recent papers [4-7] we have addressed the problem of the highenergy collision of a closed string off a configuration of parallel $\mathrm{D} p$-branes. By suitably playing with the various parameters characterizing the process, the number $N$ of branes, the energy $E$ and impact parameter $b$ of the collision, as well as the string coupling $g_{s}$, we could identify [4] a region in parameter space inside which closed string loops can be safely neglected and, consequently, there is no closed string production or gravitational bremsstrahlung, a considerable simplification. Even within this region there are several interesting regimes to study. 
There is a weak-gravity regime (corresponding to very large impact parameters compared to the other length scales in the problem) in which string-size effects can be neglected and general-relativity expectations are recovered in terms of gravitational deflection and Shapiro time delay. In this regime the resulting S-matrix satisfies elastic unitarity.

There is also a string-size-corrected weak-gravity regime (corresponding to somewhat smaller impact parameters) in which tidal excitations of the incoming closed string become important or even dominant [4]. In this regime we are still able to provide an exactly unitary S-matrix, but unitarity now works in an enlarged Hilbert space containing excited closed strings besides the incoming one. In $[5,6]$ (see also $[8,9]$ ) the microscopic structure of this S-matrix was analyzed in much detail.

There is finally a strong-gravity regime (corresponding to a gravitational radius of the effective $p$-brane geometry $R_{p}$ larger than $b$ ) in which the closed string is captured by the brane system. Physically this is the most interesting situation since, in the QFT limit, one expects information about the initial state to be lost in the capture (cf. the fall into a potential well in quantum mechanics). By contrast, string theory should again be able to give a unitary (i.e. information preserving) S-matrix by providing a microscopic description of the (open string) excitations induced on the branes by the absorbed closed string.

The study of this process is in general very hard. It can be simplified, however, under the assumption that $R_{p}$, while comparable or even larger than $b$, is still smaller than the string length parameter $l_{s}$, enhanced by a square root of the logarithm of the energy, as we will discuss in more detail in the rest of the paper. ${ }^{1}$ When this is the case the eikonal resummation of the leading terms (in energy) of the higher-order string amplitudes $[4,11,12]$ should give a correct representation of the dynamics for every value of the impact parameter, all the way down to $b=0$. Although in this limit a geometric interpretation of the brane background is lacking, the dynamics of the string-brane system remains extremely rich and interesting.

The absorption process is expected to lead to a state consisting of a very complicated (yet quantum mechanically pure) linear superposition of multi-open-string excitations of the brane system. Therefore, we may regard the problem at hand as being very close, in spirit, to the famous information puzzle arising from the formation and evaporation of a black hole from a pure initial state.

The study of a similar process was attempted before in the context of string-string collisions $[11,12]$ where the regime analogous to the one considered here, corresponds to taking the gravitational radius $R_{S} \equiv 2 G_{N} \sqrt{s}$ to be smaller than $l_{s}$ but possibly larger than $b$. Although black hole formation is not supposed to happen in such a regime, the final state was argued [11-13] to have many features in common with the one expected from an evaporating black hole. Only a rough description of the final state (basically just keeping track of the number of final strings) was obtained in [11-13]. The hope is that, in the

\footnotetext{
${ }^{1}$ In a previous paper [7] we have studied, precisely in this regime, the elastic scattering of a closed string for what concerns the resolution of the causality issue recently raised by Camanho et al. [10]. In this paper we look at a complementary aspect, the absorption of the closed string resulting in the above-mentioned production of open string excitations of the brane system.
} 
case of string-brane collisions, one should be able to go much further in the microscopic description of the final state.

Our final aim is to arrive at a unitary S-matrix describing both the tidal excitation and the absorption of the energetic incoming closed string. In this paper we shall take a first but important step in this direction by studying in detail the process at tree (i.e. disk) level. In this approximation the energetic closed string is absorbed through the formation of a single massive open string attached to the brane system. Our main result will be to derive an explicit microscopic description of this highly excited open-string state at the quantum level. In a forthcoming paper [14] a simple and intuitive explanation of the properties of this state will be given by considering a closed-string brane collision in a kinematic regime allowing for the formation of an open string at the classical level. Furthermore, when the conditions for the classical closed to open transition are not met, it will be possible to perform a semiclassical analysis which confirms qualitatively the results of this work.

The massive open string created on the branes belongs to the $n$-th level of the string spectrum, where $n \sim \alpha^{\prime} E^{2}$ is fixed by the energy of the closed string. As the energy increases we are therefore exploring higher and higher levels of the spectrum. Since the covariant methods are not very suitable to deal with generic excited states, we will work in the light-cone gauge and derive the form of the open state by taking the high-energy limit of the light-cone closed-open vertex [15-19].

The dimensionality of the Hilbert space of possible final states is exponentially large and one would expect that the massive open string would have a very complex representation in a generic basis. Instead, we find that by choosing a natural basis for the process, a light-cone gauge aligned with the direction of large momentum, detailed calculations become possible leading to an extremely simple representation of the final state.

As already mentioned, the detailed understanding of the absorption process at tree level is only the first step in the construction of a unitary S-matrix. In order to achieve this, one needs to take into account higher (open string) loops. We shall present elsewhere [20] how to generate an explicitly unitary S-matrix in a suitable narrow-resonance approximation.

This paper is organized as follows. In section 2, we first review the elastic scattering amplitude of a tachyon off a collection of D-branes and its Regge limit. We then use the eikonal and Reggeon vertex operators to derive a general formula for the Regge limit of the same type of scattering amplitude but with arbitrary external closed-string states. We finally discuss the $s$-channel factorization of the elastic string-brane amplitudes and their imaginary part, which gives the total absorption probability of a closed string by the D-branes.

In section 3 we start our analysis aiming at identifying the massive open state whose creation amplitude can account for the full absorption probability at high energy. To this purpose we introduce the closed-open transition amplitudes and the closed-open light-cone vertex. The latter is essential to work with arbitrary external closed and open states.

The form of the closed-open vertex in the high-energy limit is determined in section 4 . These results are used in section 5 to derive a simple and explicit form for the open state created on the brane system by the absorption of a closed string at high energy. We analyze in turn the absorption of a tachyon, of a massless state and of a state belonging to the 
first massive level and finally give a formula for a generic closed string. As a test of our results we show how to reconstruct the imaginary part of the elastic amplitude in impact parameter space. In section 6 we present our conclusions.

We collected some additional material in two appendices. In the first we compare the closed-open transition amplitudes given by the light-cone vertex and by the covariant methods for states belonging to the lowest levels. In the second we provide some technical details on the evaluation of the imaginary part of the disk using the closed-open vertex.

\section{Elastic scattering of closed strings by D-branes}

When a closed string propagates in the presence of a stack of $N$ coincident $\mathrm{D} p$-branes, the simplest new processes that can occur at leading order in perturbation theory are its scattering (elastic or inelastic) off the $\mathrm{D} p$-branes and its absorption by the $\mathrm{D} p$-branes. Both processes are given by a two-point function on the disk, the first with two closed string vertex operators and the second with one closed and one open string vertex operator, since at leading order in $g_{s} N$ the absorption process results in the creation of a single massive open string on the branes worldvolume. ${ }^{2}$

The possibility of having a closed-open transition induces a non-vanishing imaginary part for the tree-level elastic scattering amplitude of the closed string. The imaginary part gives the total splitting probability for the closed string to turn into an open string, a quantity that, in the high energy limit and as a function of the impact parameter $b$, will play an essential role in our analysis. Indeed, in sections 3 to 5 of our paper we will derive the explicit form of the high energy limit of the closed-open transition amplitudes, thus identifying the precise open string state created on the $\mathrm{D} p$-branes. As an important check of our result, we will verify that the square of the closed-open amplitude precisely reproduces the imaginary part of the elastic closed-closed amplitude. For this reason in this section we will derive, following the discussion in ref. [5], a general formula for the imaginary part of the elastic scattering amplitude of an arbitrary closed string off the $\mathrm{D} p$-branes in the Regge limit.

In this paper we shall restrict our attention, for simplicity, to the bosonic string. Generalization to the superstring case is in principle straightforward but is postponed to further work. We will begin this section by reviewing the elastic scattering amplitude of a closed string tachyon off a stack of $\mathrm{D} p$-branes and its imaginary part in the Regge limit as a function of the energy $s$ and the impact parameter $b$. Then, in order to obtain a formula for the total splitting probability valid for an arbitrary closed string state, we will review the results of ref. [5], where it is shown that a simple and efficient way to calculate the Regge limit of a generic elastic (or inelastic) closed-closed scattering amplitude is to evaluate the matrix elements of the eikonal operator for string-brane scattering [4]. As particular cases

\footnotetext{
${ }^{2}$ The disk amplitudes for an arbitrary number of open and closed strings with Neumann boundary conditions were first computed in ref. [21] (see eq. (3.9) of [21]). Their extension to include Dirichlet boundary conditions and their application to the physics of the D p-branes were first done in refs. [22-24]. For a more recent discussion of tree level string amplitudes involving both closed and open strings and their relations to pure open string amplitudes, which generalize the KLT relations [25], see for instance [26, 27].
} 
to illustrate this method, we will discuss two other disk amplitudes: the amplitude with two massless closed string states and the one with two massive states belonging to the first excited level of the closed string spectrum.

The disk amplitudes describing the elastic scattering of a closed string are characterized by $t$-channel poles due to the exchange of closed strings and by $s$-channel poles due to intermediate physical open strings, which will be analyzed in detail in the following sections. For instance the simplest elastic scattering amplitude, the one for the closed string tachyon, reads ${ }^{3}$

$$
A_{T T}=\frac{\kappa N T_{p}}{2} \frac{\Gamma\left(-\alpha^{\prime} s-1\right) \Gamma\left(-\frac{\alpha^{\prime}}{4} t-1\right)}{\Gamma\left(-\alpha^{\prime} s-\frac{\alpha^{\prime}}{4} t-2\right)}, \quad T_{p}=\frac{\sqrt{\pi}}{2^{4}}\left(2 \pi \sqrt{\alpha^{\prime}}\right)^{11-p}
$$

The normalization is given in terms of the gravitational coupling $\kappa^{2}=2^{-9} g_{s}^{2}(2 \pi)^{23}\left(\alpha^{\prime}\right)^{12}$, the number of branes $N$, and the brane tension $T_{p}$. The various quantities are taken for $d=26$. The Mandelstam variables are defined in terms of the external momenta $p_{1}$ and $p_{2}$ of the two closed string tachyons [22-24]

$$
\begin{aligned}
& t=-\left(p_{1}+p_{2}\right)^{2}=-4\left(E^{2}-M^{2}\right) \sin ^{2} \frac{\theta}{2}, \\
& s=-\frac{1}{4}\left(p_{1}+D p_{1}\right)^{2}=-\frac{1}{4}\left(p_{2}+D p_{2}\right)^{2}=E^{2},
\end{aligned}
$$

where $\theta$ is the angle between $\vec{p}_{1}$ and $-\vec{p}_{2}, M^{2}=-4 / \alpha^{\prime}$ and $D$ is the standard reflection matrix for a $\mathrm{D} p$-brane, $D^{\mu}{ }_{\nu}=\operatorname{diag}(1, \ldots, 1,-1, \ldots,-1)$ with the first $p+1$ eigenvalues equal to 1 and the remaining $25-p$ equal to -1 . Notice that we chose a reference frame where $\left(p_{i}+D p_{i}\right)$ has no space-like components, so $\sqrt{s}$ is equal to the energy of the closed states. Using the relation

$$
\frac{\Gamma(x)}{\Gamma(x+a)}=\sum_{n=0}^{\infty} \frac{(-1)^{n}}{\Gamma(n+1) \Gamma(a-n)} \frac{1}{x+n},
$$

with $a \neq 0,1, \ldots$, we can write the amplitude in eq. (2.1) in the form

$$
A_{T T}=\frac{\kappa N T_{p}}{2} \sum_{n=0}^{\infty} \frac{1}{n !} \frac{P_{n}\left(\frac{\alpha^{\prime} t}{4}\right)}{-\alpha^{\prime} s-1+n}, \quad P_{n}\left(\frac{\alpha^{\prime} t}{4}\right)=\frac{\Gamma\left(\frac{\alpha^{\prime}}{4} t+n+2\right)}{\Gamma\left(\frac{\alpha^{\prime}}{4} t+2\right)},
$$

that clearly displays the $s$-channel poles and their residues. There is a pole for each level of the open-string spectrum, the residue being a polynomial $P_{n}(y)$ of degree $n$ in $y=\frac{\alpha^{\prime} t}{4}$. The imaginary part of the amplitude follows from the usual $i \epsilon$ prescription and is obtained from $(2.4)$ by substituting each pole $1 /\left(-\alpha^{\prime} s+m\right)$ with $\pi i \delta\left(\alpha^{\prime} s-m\right)$

$$
\operatorname{Im} A_{T T}=\pi \frac{\kappa N T_{p}}{2} \sum_{n=0}^{\infty} \delta\left(\alpha^{\prime} s-n+1\right) \frac{1}{n !} P_{n}\left(\frac{\alpha^{\prime} t}{4}\right) .
$$

\footnotetext{
${ }^{3}$ For a derivation of this amplitude see for instance [7].
} 
In the Regge limit, $\alpha^{\prime} s \gg 1$ with $\alpha^{\prime} t$ fixed, the amplitude (2.1) behaves as ${ }^{4}$

$$
A_{T T} \sim \mathcal{A}_{T T}=\frac{\kappa N T_{p}}{2} \mathrm{e}^{-i \pi\left(1+\frac{\alpha^{\prime}}{4} t\right)} \Gamma\left(-1-\frac{\alpha^{\prime}}{4} t\right)\left(\alpha^{\prime} s\right)^{1+\frac{\alpha^{\prime}}{4} t} .
$$

Its real and imaginary part are easily evaluated

$$
\begin{aligned}
& \operatorname{Re} \mathcal{A}_{T T}=\frac{\kappa N T_{p}}{2} \cos \pi\left(1+\frac{\alpha^{\prime}}{4} t\right) \Gamma\left(-1-\frac{\alpha^{\prime}}{4} t\right)\left(\alpha^{\prime} s\right)^{1+\frac{\alpha^{\prime}}{4} t}, \\
& \operatorname{Im} \mathcal{A}_{T T}=\pi \frac{\kappa N T_{p}}{2} \frac{\left(\alpha^{\prime} s\right)^{1+\frac{\alpha^{\prime}}{4} t}}{\Gamma\left(2+\frac{\alpha^{\prime}}{4} t\right)} .
\end{aligned}
$$

It is interesting to derive the imaginary part in the Regge limit directly from the imaginary part of the full amplitude in eq. (2.5). When $\alpha^{\prime} s \gg 1$ the discrete distribution of poles can be approximated by a continuum, since the relative separation between adjacent levels of the open string spectrum that are accessible at a given energy becomes of order $\frac{1}{\alpha^{\prime} s}$. To study the contribution of the levels in a neighborhood of $\alpha^{\prime} s$ let us set

$$
n=\alpha^{\prime} s x, \quad \sum_{n=0}^{\infty} \delta\left(\alpha^{\prime} s-n+1\right) \sim \int_{0}^{\infty} d x \delta\left(1-x+\frac{1}{\alpha^{\prime} s}\right) .
$$

In the limit $\alpha^{\prime} s \gg 1$ we then reproduce

$$
\begin{aligned}
\operatorname{Im} A_{T T} & \sim \pi \frac{\kappa N T_{p}}{2} \int_{0}^{\infty} d x \delta(1-x) \frac{P_{n}\left(\frac{\alpha^{\prime} t}{4}\right)}{\Gamma(n+1)} \sim \pi \frac{\kappa N T_{p}}{2} \int_{0}^{\infty} d x \delta(1-x) \frac{\left(\alpha^{\prime} s x\right)^{1+\frac{\alpha^{\prime} t}{4}}}{\Gamma\left(2+\frac{\alpha^{\prime} t}{4}\right)} \\
& =\pi \frac{\kappa N T_{p}}{2} \frac{\left(\alpha^{\prime} s\right)^{1+\frac{\alpha^{\prime}}{4} t}}{\Gamma\left(2+\frac{\alpha^{\prime}}{4} t\right)} .
\end{aligned}
$$

In the following we will also be interested in scattering and absorption processes happening at fixed impact parameter. The imaginary part of the elastic disk amplitude in impact parameter space is

$$
\operatorname{Im} \mathcal{A}_{T T}(s, b)=\int \frac{d^{24-p} q}{(2 \pi)^{24-p}} e^{-i \vec{b} \vec{q}} \operatorname{Im} \mathcal{A}_{T T}(s, t) \sim \pi \frac{\kappa N T_{p}}{2} \frac{\alpha^{\prime} s}{\left(\pi \alpha^{\prime} \log \alpha^{\prime} s\right)^{\frac{24-p}{2}}} e^{-\frac{b^{2}}{\alpha^{\prime} \log \alpha^{\prime} s}},
$$

where $\vec{q}$ is a $24-p$-dimensional vector satisfying $-(\vec{q})^{2} \equiv t$. In the last step we approximated $\Gamma\left(2+\frac{\alpha^{\prime}}{4} t\right) \sim 1$. This approximation amounts to neglecting at any given order in an expansion in powers of $\frac{b^{2}}{\alpha^{\prime} \log \alpha^{\prime} s}$ terms that are suppressed by additional powers of $\log \alpha^{\prime} s$. The imaginary part has a characteristic Gaussian dependence on the impact parameter, that indicates that in the Regge limit the $\mathrm{D} p$-branes behave as black disks with a radius growing like the square root of the logarithm of the energy, $R \sim \sqrt{\alpha^{\prime} \log \alpha^{\prime} s}$.

The elastic amplitude for the tachyon is extremely compact due to the fact that this state is a scalar with a simple vertex operator

$$
\mathcal{V}_{T, \bar{T}}=\frac{\kappa}{2 \pi} e^{i p X}
$$

\footnotetext{
${ }^{4}$ Here and in the following we will use the symbol $\mathcal{A}$ for the Regge limit of an amplitude $A$.
} 
The vertex operator for a generic closed string state $|\psi\rangle$ can be written as follows:

$$
\mathcal{V}_{S, \bar{S}}=\frac{\kappa}{2 \pi} \epsilon_{\mu_{1} \ldots \mu_{r}} \bar{\epsilon}_{\nu_{1} \ldots \nu_{s}} \mathrm{~V}_{S}^{\mu_{1} \ldots \mu_{r}} \overline{\mathrm{V}}_{\bar{S}}^{\nu_{1} \ldots \nu_{s}}
$$

where $S$ and $\bar{S}$ are labels that identify the little group representations of the left and right part of the closed state, $\epsilon, \bar{\epsilon}$ the corresponding polarization tensors and ${ }^{5}$

$$
\mathrm{V}_{S}=\operatorname{Pol}_{S}\left(\partial^{r} X\right) e^{i p X_{L}},
$$

with $\mathrm{Pol}_{S}$ a polynomial in the holomorphic derivatives of $X^{\mu}$. Elastic and inelastic amplitudes for arbitrary states of the string spectrum become more and more complex as the number of possible contractions between momenta and polarizations increases.

Their structure however drastically simplifies in the Regge limit and, as discussed in [5], it is possible to give a general and explicit formula in terms of the matrix elements of the phase of the eikonal operator $[4,11,12]$. The Regge limit is characterized by a single spatial direction of large momentum that naturally leads to a separation of the dynamics in a longitudinal and a transverse part. It is therefore convenient to introduce a frame consisting of two light-like vectors $e^{ \pm}$, whose spatial component coincides with the direction of large momentum and that satisfy the following conditions:

$$
e^{+} \cdot e^{+}=e^{-} \cdot e^{-}=0, \quad e^{+} \cdot e^{-}=1,
$$

together with 24 transverse spatial vectors $e^{i}$, orthogonal to $e^{ \pm}$. By convention we will choose the large momentum along the spatial axis corresponding to the last coordinate

$$
e^{+}=\frac{1}{\sqrt{2}}(-1,0, \ldots, 0,1), \quad e^{-}=\frac{1}{\sqrt{2}}(1,0, \ldots, 0,1) .
$$

When $\alpha^{\prime} s \gg 1$ and $\alpha^{\prime} t$ is kept fixed, the scattering process is dominated by the exchange of the states of the leading Regge trajectory that can be represented by a single effective string state, the Reggeon [28-30]

$$
\mathrm{V}_{R}=\left(\sqrt{\frac{2}{\alpha^{\prime}}} \frac{i \partial X^{+}}{\sqrt{\alpha^{\prime}} E}\right)^{1+\frac{\alpha^{\prime} t}{4}} e^{-i q X_{L}}, \quad X^{+}=e^{+} \cdot X .
$$

In this limit the string-brane scattering amplitudes factorize in the product of the threepoint coupling of the two external states to the Reggeon and the one-point function of the Reggeon in the brane background, which coincides with the Regge limit of the tachyon scattering amplitude, eq. (2.6). If the initial state is $\left(S_{1}, \bar{S}_{1}\right)$ with polarization tensor $\epsilon, \bar{\epsilon}$ and the final state $\left(S_{2}, \bar{S}_{2}\right)$ with polarization tensor $\zeta, \bar{\zeta}$ we can write

$$
\mathcal{A}_{\left(S_{1}, \bar{S}_{1}\right),\left(S_{2}, \bar{S}_{2}\right)}=\mathcal{A}_{T T} C_{S_{1}, S_{2}, R} \bar{C}_{\bar{S}_{1}, \bar{S}_{2}, \bar{R}}
$$

where

$$
C_{S_{1}, S_{2}, R}=\left\langle\mathrm{V}_{S_{1}} \mathrm{~V}_{S_{2}} \mathrm{~V}_{R}\right\rangle=\epsilon_{\mu_{1} \ldots \mu_{r}} \zeta_{\nu_{1} \ldots \nu_{s}} T_{S_{1}, S_{2}, R}^{\mu_{1} \ldots \mu_{r} ; \nu_{1} \ldots \nu_{s}}
$$

\footnotetext{
${ }^{5}$ We write $X(z, \bar{z})=X_{L}(z)+X_{R}(\bar{z})$.
} 
The tensors $T_{S_{1}, S_{2}, R}$ are formed using the flat metric $\eta^{\mu \nu}$, the momentum transfer $q^{\mu}$ and the longitudinal polarization vector $v^{\mu}$, with coefficients that depend only on $t$ and the masses of the external states (see [5] for details).

The formula (2.17) can be simplified even further if we describe the string spectrum using a basis of DDF operators rather than a basis of covariant vertex operators [5]. In this way we only maintain manifest invariance with respect to the transverse $\mathrm{SO}(24)$ rotation group but it becomes straightforward to enumerate the physical states. Moreover the couplings of the external states to the Reggeon become elementary and all the treelevel scattering amplitudes in the Regge limit can be represented as matrix elements of a very simple operator $W(s, q)$, closely related to the phase $\hat{\delta}(s, q)$ of the eikonal operator. These simplifications occur if the null vector required by the DDF construction is chosen proportional to $e^{+}$. In this way the amplitudes obtained using the DDF operators coincide with the amplitudes given by the three-string vertex in the light-cone gauge adapted to the kinematics of the Regge limit, as given in eq. (2.15).

The operator $W$ that gives the tree-level scattering amplitudes is ${ }^{6}$

$$
W(s, q)=4 E \hat{\delta}(s, q)=\mathcal{A}_{T T}(s, t) \int_{0}^{2 \pi} \frac{d \sigma}{2 \pi}: e^{i q \hat{X}}: .
$$

The operators $X^{i}$ are the string position operators (without zero modes and at $\tau=0$ )

$$
\hat{X}^{i}(\sigma)=i \sqrt{\frac{\alpha^{\prime}}{2}} \sum_{k \neq 0} \frac{1}{k}\left(A_{k}^{i} e^{-i k \sigma}+\bar{A}_{k}^{i} e^{i k \sigma}\right),
$$

in a light-cone gauge with the spatial direction aligned with the direction of large momentum. The evaluation of the Regge limit of elastic or inelastic scattering amplitudes become straightforward using the operator in eq. (2.19). We illustrate this method with the elastic scattering of states in the massless and in the first massive levels. A generic massless state (level $N_{c}=1$ ) can be written as follows:

$$
\left|g_{\epsilon}, \bar{g}_{\bar{\epsilon}}\right\rangle=\epsilon_{i} \bar{\epsilon}_{j} A_{-1}^{i} \bar{A}_{-1}^{j}|0\rangle
$$

The polarization tensor

$$
\epsilon_{i j}=\epsilon_{i} \bar{\epsilon}_{j},
$$

can be decomposed in a trace, symmetric traceless and antisymmetric part corresponding respectively to the dilaton, graviton and Kalb-Ramond field. Denoting with $\epsilon$ the polarization of the incoming state and with $\zeta$ the polarization of the outgoing state we find

$$
\mathcal{A}_{g g}=\left\langle g_{\zeta}, \bar{g}_{\bar{\zeta}}|W(s, q)| g_{\epsilon}, \bar{g}_{\bar{\epsilon}}\right\rangle=\left(\epsilon \zeta-\frac{\alpha^{\prime}}{2}(\epsilon q)(\zeta q)\right)\left(\bar{\epsilon} \bar{\zeta}-\frac{\alpha^{\prime}}{2}(\bar{\epsilon} q)(\bar{\zeta} q)\right) \mathcal{A}_{T T} .
$$

This is therefore the Regge limit of the disk scattering amplitude involving two massless closed strings. $^{7}$ The structure of the asymptotic form of this amplitude, eq. (2.23), is a

\footnotetext{
${ }^{6}$ See [7] for a derivation of the eikonal operator for string-brane scattering in the bosonic string.

${ }^{7} \mathrm{~A}$ derivation of the complete scattering amplitude involving two massless closed strings, and not just of its Regge limit, can be found in section 2 and appendix A of ref. [7] and it agrees with the one given in ref. [22] for the case of the bosonic string.
} 
general result. In the Regge limit all the scattering amplitudes are obtained by multiplying the tachyon amplitude with a suitable polynomial in the polarizations and the momentum transfer. In impact parameter space the polynomial becomes a differential operator in $\partial_{b_{i}}$ acting on $\mathcal{A}_{T T}(s, b)$. For instance

$$
\mathcal{A}_{g g}(s, \vec{b})=\left(\epsilon \zeta+\frac{\alpha^{\prime}}{2}\left(\epsilon \partial_{b}\right)\left(\zeta \partial_{b}\right)\right)\left(\bar{\epsilon} \bar{\zeta}+\frac{\alpha^{\prime}}{2}\left(\bar{\epsilon} \partial_{b}\right)\left(\bar{\zeta} \partial_{b}\right)\right) \mathcal{A}_{T T}(s, b) .
$$

The differential operator reflects the presence of higher derivative corrections to the gravitational couplings in the bosonic string $[7,10]$. As far as the imaginary part is concerned the derivatives generate terms suppressed by additional powers of $\log \left(\alpha^{\prime} s\right)$ and we will neglect them for consistency with the approximations made in deriving eq. (2.10). Therefore

$$
\operatorname{Im} \mathcal{A}_{g g}(s, \vec{b}) \sim \epsilon \zeta \bar{\epsilon} \bar{\zeta} \operatorname{Im} \mathcal{A}_{T T}(s, b) .
$$

As an additional example of the general structure of the amplitudes let us consider the first massive level, that contains the $\mathrm{SO}(25)$ representations in the tensor product of two symmetric traceless tensors, one for the left and one for the right half of the string. The $\mathrm{SO}(25)$ symmetric traceless tensor decomposes into a symmetric tensor and a vector of the transverse $\mathrm{SO}(24)$

$$
\left|H_{\epsilon}\right\rangle=\frac{1}{\sqrt{2}} \epsilon_{i j} A_{-1}^{i} A_{-1}^{j}|0\rangle, \quad\left|L_{\epsilon}\right\rangle=\frac{1}{\sqrt{2}} \epsilon_{i} A_{-2}^{i}|0\rangle .
$$

The symmetric tensor can be further decomposed into a traceless and a trace part. Let us discuss separately the elastic scattering of a closed state given by the product of two tensors $(H \otimes \bar{H})$ and by the product of two vectors $(L \otimes \bar{L})$. The remaining cases $(H \otimes \bar{L}$ and $L \otimes \bar{H}$ ) can be analyzed in a similar way. For the state

$$
\left|H_{\epsilon}, \bar{H}_{\epsilon}\right\rangle=\frac{1}{2} \epsilon_{i j} \bar{\epsilon}_{k l} A_{-1}^{i} A_{-1}^{j} \bar{A}_{-1}^{k} \bar{A}_{-1}^{l}|0\rangle,
$$

we find

$$
\begin{aligned}
\mathcal{A}_{H H}= & \left(\epsilon_{i j} \zeta_{i j}-\frac{\alpha^{\prime}}{2} \epsilon_{i i^{\prime}} q^{i^{\prime}} \zeta_{j j^{\prime}} q^{j^{\prime}}+\frac{\alpha^{\prime 2}}{16} \epsilon_{i j} q^{i} q^{j} \zeta_{i^{\prime} j^{\prime}} q^{i^{\prime}} q^{j^{\prime}}\right) \\
& \left(\bar{\epsilon}_{k l} \bar{\zeta}_{k l}-\frac{\alpha^{\prime}}{2} \bar{\epsilon}_{k k^{\prime}} q^{k^{\prime}} \bar{\zeta}_{l l^{\prime}} q^{l^{\prime}}+\frac{\alpha^{\prime 2}}{16} \bar{\epsilon}_{k l} q^{k} q^{l} \bar{\zeta}_{k^{\prime} l^{\prime}} q^{k^{\prime}} q^{l^{\prime}}\right) \mathcal{A}_{T T} .
\end{aligned}
$$

For the state

we find

$$
\left|L_{\epsilon}, \bar{L}_{\epsilon}\right\rangle=\frac{1}{2} \epsilon_{i} \bar{\epsilon}_{j} A_{-2}^{i} \bar{A}_{-2}^{j}|0\rangle,
$$

$$
\mathcal{A}_{L L}=\left(\epsilon \zeta-\frac{\alpha^{\prime}}{8}(\epsilon q)(\zeta q)\right)\left(\bar{\epsilon} \bar{\zeta}-\frac{\alpha^{\prime}}{8}(\bar{\epsilon} q)(\bar{\zeta} q)\right) \mathcal{A}_{T T} .
$$

A generic closed state $|\psi\rangle$ at level $N_{c}$ is characterized by the collection of left and right modes that create it acting on the vacuum

$$
A_{-k_{\alpha}}^{i_{\alpha}}, \quad \bar{A}_{-\bar{k}_{\beta}}^{i_{\beta}}, \quad \sum_{\alpha} k_{\alpha}=\sum_{\beta} \bar{k}_{\beta}=N_{c},
$$


and by a collection of polarization vectors $\epsilon_{\alpha}^{i_{\alpha}}, \bar{\epsilon}_{\beta}^{i_{\beta}}$. To describe a state transforming in a specific irreducible representation of the transverse $\mathrm{SO}(24)$, one simply acts with the corresponding Young projector on the tensor product of the vector representations. The elastic scattering amplitude

$$
\mathcal{A}_{\psi_{\zeta} \psi_{\epsilon}}=\mathcal{A}_{T T} \int_{0}^{2 \pi} \frac{d \sigma}{2 \pi}\left\langle\psi_{\zeta}\left|: e^{i q X}:\right| \psi_{\epsilon}\right\rangle
$$

can be evaluated by expanding the exponential and collecting the terms that give a nonvanishing matrix element. ${ }^{8}$ This will result in a polynomial where the polarization vectors of the incoming and outgoing states are contracted among themselves or with the momentum transfer, like in the examples just discussed. From (2.32) it is clear that at $t=0$ the elastic amplitude reduces to the contraction between the initial and final polarization tensors

$$
\mathcal{A}_{\psi_{\zeta} \psi_{\epsilon}}(s, 0)=\prod_{\alpha}\left(\zeta_{\alpha} \epsilon_{\alpha}\right) \prod_{\beta}\left(\bar{\zeta}_{\beta} \bar{\epsilon}_{\beta}\right) \mathcal{A}_{T T}(s, 0)=\pi \frac{\kappa N T_{p}}{2} \alpha^{\prime} s \prod_{\alpha}\left(\zeta_{\alpha} \epsilon_{\alpha}\right) \prod_{\beta}\left(\bar{\zeta}_{\beta} \bar{\epsilon}_{\beta}\right) .
$$

In impact parameter space the polynomial in the momentum transfer that multiplies the tachyon amplitude $\mathcal{A}_{T T}(s, t)$ becomes a differential operator in $\partial_{b_{i}}$ that acts on $\mathcal{A}_{T T}(s, b)$. Up to terms suppressed by additional powers of $\log \alpha^{\prime} s$, the imaginary part is simply

$$
\operatorname{Im} \mathcal{A}_{\psi_{\zeta} \psi_{\epsilon}}(s, b)=\prod_{\alpha}\left(\zeta_{\alpha} \epsilon_{\alpha}\right) \prod_{\beta}\left(\bar{\zeta}_{\beta} \bar{\epsilon}_{\beta}\right) \operatorname{Im} \mathcal{A}_{T T}(s, b)
$$

To summarize, in this section we have reviewed the computation of the Regge limit of the disk scattering amplitudes involving two arbitrary closed string states [5]. It turns out that, in the Regge limit, all the scattering amplitudes are obtained by multiplying the tachyon amplitude with a suitable polynomial in the polarizations and the momentum transfer. We have seen that these amplitudes have $s$-channel poles for values of $s$ such that $1+\alpha^{\prime} s=n \geq 0$ corresponding to the creation of an open string. The residue of the pole, given by the imaginary part of the amplitude, is equal to the absolute value square of the coupling of the closed string with a specific open string state belonging to the level $n$. The aim of the next three sections will be to find a precise and explicit form for this highly excited open string state.

\section{Absorption of closed strings by D-branes}

In the background of a $\mathrm{D} p$-brane a closed string can split turning itself into an open string. This process is described at tree level by a two-point function on the disk with one closed and one open vertex operator. These closed-open correlation functions give the transition amplitude to specific open string states while the imaginary part of the elastic amplitude, discussed in the previous section, gives the total splitting probability. In order to derive

\footnotetext{
${ }^{8} \mathrm{In}[5]$ it is shown in detail how to relate the matrix elements of the eikonal operator and the covariant amplitudes.
} 
the quantum state that represents the massive open string we need to determine the high energy limit of the closed-open couplings for arbitrary external states.

We begin this section by discussing the closed-open amplitudes in the basis of the covariant vertex operators. Since the covariant methods are not very suitable to study highly excited string states, we then introduce the light-cone closed-open vertex [15-19] that encodes all the correlation functions between arbitrary closed and open string states. This vertex can be derived by a variety of methods: factorizing the non-planar one-loop two open-string amplitude $[15,16]$, finding the operator solution to the continuity conditions for the string coordinates [17, 19] or evaluating the string path integral [18]. It can also be derived using the DDF operators, following the analysis in [31] for the three openstring vertex.

In this section we will give the explicit form of the vertex for a generic $\mathrm{D} p$-brane background and discuss two inequivalent choices for the spatial axis of the light-cone: along the branes worldvolume or along the direction of collision. The latter choice will turn out to be the most convenient to derive the high energy limit of the vertex. After discussing this limit in section 4 , we will be able to determine in section 5 the precise form of the highly excited open state and verify that it reproduces the imaginary part of the elastic amplitudes at high energy.

\subsection{Closed-open amplitudes}

Let us consider the transition from a closed string at level $N_{c}$ with spatial momentum $\vec{p}_{c}$ in a direction orthogonal to the branes ${ }^{9}$

$$
p_{c}=\left(E, \overrightarrow{0}_{p}, \vec{p}_{c}\right), \quad \alpha^{\prime} M^{2}=\alpha^{\prime}\left(E^{2}-\vec{p}_{c}^{2}\right)=4\left(N_{c}-1\right) \quad N_{c}=0,1,2 \ldots,
$$

to an open string at level $n$ with momentum

$$
p_{o}=\left(-m, \overrightarrow{0}_{p}, \overrightarrow{0}_{25-p}\right), \quad \alpha^{\prime} m^{2}=-\alpha^{\prime} p_{o}^{2}=n-1 \quad n=0,1,2 \ldots .
$$

The transition is possible if

$$
\alpha^{\prime} E^{2}=\alpha^{\prime} m^{2}=n-1, \quad \alpha^{\prime} \vec{p}_{c}^{2}=n-4 N_{c}+3 \geq 0 .
$$

The lowest accessible level is therefore $n=4 N_{c}-3$. As the momentum of the closed string increases it is possible to reach an arbitrarily high level of the open string spectrum. For instance the tachyon can create any open state, the massless states can create an open string with $n \geq 2$, closed states from the first massive level can create an open string with $n=5$ and above. For the open vertex operators we use a notation similar to the one introduced in eq. (2.12)

$$
\mathcal{V}_{\chi}=g_{o} \epsilon_{\mu_{1} \ldots \mu_{r}} \mathrm{~V}_{\chi}^{\mu_{1} \ldots \mu_{r}}
$$

where $g_{o}=\sqrt{2 \alpha^{\prime}} g_{p+1}$, with $g_{p+1}$ the coupling constant of the gauge theory living on the branes world-volume. ${ }^{10}$ When we have a stack of $N$ D -branes, the open state carries

\footnotetext{
${ }^{9}$ The components of the momenta are given in the following order: $(t, v o l, p e r p)$, where $t$ is the time direction, vol the $p$-dimensional branes volume, perp the $(25-p)$-dimensional space transverse to the branes.

${ }^{10}$ In terms of the string coupling constant $g_{s}$ and the Regge slope $\alpha^{\prime}$ this constant is given by $g_{p+1}^{2}=$ $2 \pi g_{s}\left(2 \pi \sqrt{\alpha^{\prime}}\right)^{p-3}$. The Chan-Paton factors are normalized as $\operatorname{Tr}\left(\lambda^{i} \lambda^{j}\right)=\delta^{i j}$.
} 
Chan-Paton factors $\lambda^{a}$ that form a basis for the Lie algebra of $\mathrm{U}(1) \times \mathrm{SU}(N)$. Since the closed strings are singlets, the open string state must belong to the $\mathrm{U}(1)$ factor with $\lambda_{s}=\frac{1}{\sqrt{N}} \mathbb{I}_{N}$ and $\operatorname{tr}\left(\lambda_{s}\right)=\sqrt{N}$. At tree level the absorption amplitude is given by the correlation function on the disk of two vertex operators, one for the closed string state $\psi$ and one for the open string state $\chi$

$$
B_{\psi, \chi}\left(p_{c}\right)=\beta\left\langle\mathrm{V}_{S} \mathrm{~V}_{\bar{S}} \mathrm{~V}_{\chi}\right\rangle_{D}
$$

where the normalization $\beta$ is given by

$$
\beta^{2}=\frac{\kappa}{2 \alpha^{\prime}} N T_{p}
$$

The imaginary part of the two-point elastic closed string amplitudes can be written as

$$
\operatorname{Im} \mathcal{A}_{\psi, \psi}\left(p_{1}, p_{2}\right)=\pi \alpha^{\prime} \sum_{\chi} B_{\psi, \chi}\left(p_{1}\right) B_{\psi, \chi}^{*}\left(p_{2}\right) .
$$

The sum extends to all the open states $\chi$ at level $n$ and includes a sum over their polarizations. Some explicit examples of closed-open amplitudes obtained by evaluating the correlation functions on the disk can be found in appendix A. Since we are interested in the absorption of highly energetic strings and therefore in very massive open states, the direct evaluation of correlation functions of vertex operators becomes soon prohibitively complex. We would like to have a simple method to derive the closed-open amplitudes when the strings are highly energetic and to characterize the resulting open string state that reproduces eq. (3.7). We will develop this method in the following sections by studying the high-energy limit of the light-cone closed-open string vertex, to which we now turn.

\subsection{The closed-open string vertex}

The closed-open light-cone vertex describes the transition from an arbitrary closed string state to an arbitrary open state living on a $\mathrm{D} p$-brane. The closed-open vertex for the bosonic string for the case of purely Neumann boundary conditions was first discussed in $[15,16]$ and then generalized to include ghosts [18] and purely Dirichlet boundary conditions [19]. The extension to the superstring in the Green-Schwarz formalism was considered in [17]. Here we give the explicit form of the vertex, which we derived using the DDF operators as in [31], for a generic D $p$-brane background. Indeed, by observing that the vertex operator of a closed string is the product of two vertex operators of open strings, the closed-open vertex can be easily deduced from the three open-string vertex of [31], as it will be clear from the form of the vertex displayed below.

The light-cone is determined by two light-like vectors $e^{ \pm}$satisfying the following conditions:

$$
e^{+} \cdot e^{+}=e^{-} \cdot e^{-}=0, \quad e^{+} \cdot e^{-}=1 .
$$

There are three inequivalent choices for the spatial direction of the light-cone: along the direction of the large momentum carried by the closed string, along the volume of the $\mathrm{D} p$-branes or transverse to both the branes and the direction of large momentum. We will 
discuss explicitly the first two choices. As we will see, the open state created at high energy has an extremely simple description if the light-cone is chosen along the direction of large momentum, as we did in the previous section in our discussion of the Regge limit of the closed-closed scattering amplitudes and their relation to the eikonal operator.

Having chosen $e^{ \pm}$the light-cone vertex is given by

$$
\left|V_{B}\right\rangle=\beta \exp \left[\sum_{r, s=1}^{3} \sum_{k, l=1}^{\infty} \frac{1}{2} A_{-k}^{r, i} N_{k l}^{r s} A_{-l}^{s, i}+\sum_{r=1}^{3} \sum_{k=1}^{\infty} P^{i} N_{k}^{r} A_{-k}^{r, i}\right] \prod_{r=1}^{3}\left(|0\rangle^{(r)}\right),
$$

where

$$
P_{i} \equiv \sqrt{2 \alpha^{\prime}}\left[\alpha_{r} p_{i}^{(r+1)}-\alpha_{r+1} p_{i}^{(r)}\right] .
$$

Let us explain our notation. The normalization constant $\beta$ is given in eq. (3.6) and it is related to the normalization of the closed 2-point function on the disk. The index $i$ runs along the $d-2$ directions orthogonal to both $e^{ \pm}(d=26$ for the bosonic string) and the quantities labelled by $r, s=1,2$ refer to the left/right parts of the closed string, while those labelled by $r, s=3$ refer to the open state. In particular

$$
p^{(1)}=\frac{p_{c}}{2}, \quad p^{(2)}=\frac{D p_{c}}{2}, \quad p^{(3)}=p_{o},
$$

where $p_{c}$ is the momentum of the closed string ${ }^{11}$

$$
p_{c}=\left(E, \overrightarrow{0}_{p}, \vec{p}_{t}, p\right), \quad \alpha^{\prime} M^{2}=-\alpha^{\prime} p_{c}^{2}=4\left(N_{c}-1\right),
$$

and $p_{o}$ the momentum of the open string

$$
p_{o}=\left(-m, \overrightarrow{0}_{p}, \overrightarrow{0}_{24-p}, 0\right), \quad \alpha^{\prime} m^{2}=-\alpha^{\prime} p_{o}^{2}=n-1 .
$$

Finally, the harmonic oscillators satisfy the following commutation relations:

$$
\left[A_{k}^{r, i}, A_{h}^{s, j}\right]=k \delta_{h+k, 0} \delta^{r s} \delta^{i j} .
$$

When considering the high energy limit we will choose, as in the previous section, the large momentum along the spatial axis corresponding to the last coordinate, i.e. $p^{2} \gg \vec{p}_{t}^{2}$. We will use capital letters for the light-cone modes of the closed string and small case letters for the light-cone modes of the open string

$$
A_{k}^{1, i}=A_{k}^{i}, \quad A_{k}^{2, i}=\left(D \bar{A}^{i}\right)_{k}, \quad A_{k}^{3, i}=a_{k}^{i} .
$$

Finally the Neumann matrices $N$ in the vertex are

$$
\begin{aligned}
& N_{k}^{r}=-\frac{1}{k \alpha_{r+1}}\left(\begin{array}{c}
-k \frac{\alpha_{r+1}}{\alpha_{r}} \\
k
\end{array}\right)=\frac{1}{\alpha_{r} k !} \frac{\Gamma\left(-k \frac{\alpha_{r+1}}{\alpha_{r}}\right)}{\Gamma\left(-k \frac{\alpha_{r+1}}{\alpha_{r}}+1-k\right)}, \\
& N_{k l}^{r s}=-\frac{k l \alpha_{1} \alpha_{2} \alpha_{3}}{k \alpha_{s}+l \alpha_{r}} N_{k}^{r} N_{l}^{s}=-\frac{\alpha_{1} \alpha_{2} \alpha_{3}}{k \alpha_{s}+l \alpha_{r}} \frac{1}{\alpha_{r+1} \alpha_{s+1}}\left(\begin{array}{c}
-k \frac{\alpha_{r+1}}{\alpha_{r}} \\
k
\end{array}\right)\left(\begin{array}{c}
-l \frac{\alpha_{s+1}}{\alpha_{s}} \\
l
\end{array}\right),
\end{aligned}
$$

\footnotetext{
${ }^{11}$ The components of the momenta are given in the following order: $(t, v o l, p e r p, z)$, where $t$ is the time direction, vol the $p$-dimensional branes volume, perp the $(24-p)$-dimensional space transverse to the branes and to the direction $z$ of the large momentum $p$.
} 
while the quantities $\alpha_{r}$ are given by

$$
\alpha_{r}=2 \sqrt{2 \alpha^{\prime}}\left(e^{+} p^{(r)}\right), \quad r=1,2,3,
$$

with

$$
\alpha_{1}+\alpha_{2}+\alpha_{3}=0
$$

The momenta $P_{i}$ and the light-cone components $\alpha_{i}$ depend on the choice of gauge. We give below the explicit expressions for two inequivalent choices of the spatial components of the light-cone vectors $e^{ \pm}$: along the direction of the large momentum carried by the closed string and along the worldvolume of the $\mathrm{D} p$-branes.

The first choice can be made for every $\mathrm{D} p$-brane background. The light-cone vectors are

$$
e^{+}=\frac{1}{\sqrt{2}}(-1,0, \ldots, 0,1), \quad e^{-}=\frac{1}{\sqrt{2}}(1,0, \ldots, 0,1),
$$

and the $\alpha_{r}$ are equal to

$$
\begin{aligned}
& \alpha_{1}=\sqrt{\alpha^{\prime}}(E+p)=\sqrt{n-1}+\sqrt{n-1-4 \omega}, \\
& \alpha_{2}=\sqrt{\alpha^{\prime}}(E-p)=\sqrt{n-1}-\sqrt{n-1-4 \omega}, \\
& \alpha_{3}=-2 \sqrt{\alpha^{\prime}} E=-2 \sqrt{n-1},
\end{aligned}
$$

where

$$
\omega=\frac{\alpha^{\prime}}{4}\left(M^{2}+\vec{p}_{t}^{2}\right)=N_{c}-1+\frac{\alpha^{\prime} \vec{p}_{t}^{2}}{4} .
$$

In this light-cone gauge the index $i$ runs over the directions transverse to the branes and to the direction of large momentum, i.e. $i=p+1, \ldots, 24$. Using eq. (3.11) we find

$$
P_{i}=\alpha_{3} \sqrt{\frac{\alpha^{\prime}}{2}} p_{t, i}
$$

Note that the argument of the Gamma functions in the Neumann coefficients depends on the value of the energy and of the transverse momentum.

The second gauge choice is possible only for $\mathrm{D} p$-branes with $p \geq 1$. The light-cone vectors are

$$
e^{+}=\frac{1}{\sqrt{2}}(-1,1, \ldots, 0,0), \quad e^{-}=\frac{1}{\sqrt{2}}(1,1, \ldots, 0,0) .
$$

We then find

$$
\alpha_{1}=\alpha_{2}=\sqrt{\alpha^{\prime}} E, \quad \alpha_{3}=-2 \sqrt{\alpha^{\prime}} E
$$

and

$$
P_{i}=\alpha_{3} \sqrt{\frac{\alpha^{\prime}}{2}} p_{c, i}
$$

where $p_{c, i}$ are the components of the momentum of the closed string along the transverse directions. In this light-cone gauge the index $i$ runs over all directions transverse to the brane, $i=p+1, \ldots, 25$. In this case the argument of the Gamma functions in the Neumann coefficients does not depend on the value of the energy and of the transverse momentum. 
In the rest of this paper we will work mostly in the first gauge, where we will find a very simple representation for the massive open string. Before continuing, there is a subtlety related to this gauge choice for the open string that deserves some explanations. The gauge choice $X^{+}=2 \alpha^{\prime} p^{+} \tau$ together with the Virasoro constraints requires that the two open string coordinates chosen to form the light-cone satisfy Neumann boundary conditions. This seems incompatible with defining the coordinates $X^{ \pm}$for the open string states as the combination of the time coordinate $X^{0}$, which satisfies Neumann boundary conditions, and the direction of collision $Z$, which satisfies Dirichlet boundary conditions. For instance, for any given $X^{0}$ and $Z$ the reparametrization of the open worldsheet required to set $X^{+}=2 \alpha^{\prime} p^{+} \tau$ does not leave the worldsheet boundaries fixed.

The way around this problem is to define a modified light-cone gauge using for the open string instead of the coordinate $Z$ a coordinate $\tilde{Z}$ given by ${ }^{12}$

$$
Z(\tau, \sigma)=z\left(\sigma^{+}\right)-z\left(\sigma^{-}\right) \quad \mapsto \quad \tilde{Z}(\tau, \sigma)=z\left(\sigma^{+}\right)+z\left(\sigma^{-}\right),
$$

where $\sigma^{ \pm}=\tau \pm \sigma$. The coordinate $\tilde{Z}$ satisfies Neumann boundary conditions and there is no subtlety in fixing the light-cone gauge $\tilde{X}^{+}=2 \alpha^{\prime} \tilde{p}^{+} \tau$, where $\tilde{X}^{ \pm}=\frac{1}{\sqrt{2}}\left(X^{0} \pm \tilde{Z}\right)$ and $\sqrt{2} \tilde{p}^{+}=p_{o}^{0}$. Since the massive open strings do not have a momentum zero-mode, their description in terms of the coordinate $Z$ or the coordinate $\tilde{Z}$ is equivalent. ${ }^{13}$

In eq. (3.9) we wrote the vertex as a ket in the closed-open Hilbert space. Equivalently, we could represent it as an operator mapping the closed string Hilbert space to the open string Hilbert space, simply by replacing all the closed string creation operators with annihilation operators. Given a closed state $|\psi\rangle$ at level $N_{c}$ and with energy $\alpha^{\prime} E^{2}=n-1$, the closed-open vertex gives its couplings to all the open string states $|\chi\rangle$ at level $n$

$$
B_{\psi \chi}=\langle\chi|V| \psi\rangle \text {. }
$$

The imaginary part of the elastic disk amplitude with two external closed string states $\left|\psi_{1}\right\rangle$ and $\left|\psi_{2}\right\rangle$ can be computed combining two closed-open vertices. At finite energy it is convenient to choose in our first light-cone gauge a brick-wall frame where the momenta of the incoming and outgoing closed strings are given by

$$
p_{1}=\left(E, \overrightarrow{0}_{p}, \frac{\vec{q}}{2}, p\right), \quad p_{2}=\left(-E, \overrightarrow{0}_{p}, \frac{\vec{q}}{2},-p\right),
$$

so that $t=-\left(p_{1}+p_{2}\right)^{2}=-\vec{q}^{2}$. The angle $\theta$ between $\vec{p}_{1}$ and $-\vec{p}_{2}$ is given by

$$
\frac{\vec{q}^{2}}{4}=\left(E^{2}-M^{2}\right) \sin ^{2} \frac{\theta}{2}, \quad p^{2}=\left(E^{2}-M^{2}\right) \cos ^{2} \frac{\theta}{2} .
$$

We can then write

$$
\operatorname{Im} A_{\psi \psi}(s, t)=\pi \alpha^{\prime}\left\langle\psi_{2}\left|V_{\vec{q} / 2}^{\dagger} V_{\vec{q} / 2}\right| \psi_{1}\right\rangle, \quad \alpha^{\prime} s=n-1, \quad \alpha^{\prime} t=-\alpha^{\prime} \vec{q}^{2} .
$$

\footnotetext{
${ }^{12}$ We write $X(\tau, \sigma)=X_{L}\left(\sigma^{+}\right)+X_{R}\left(\sigma^{-}\right)$.

${ }^{13}$ In terms of the original coordinates this gauge choice sets $X_{L}^{+}\left(\sigma^{+}\right)=\alpha^{\prime} p_{L}^{+} \sigma^{+}$and $X_{R}^{-}\left(\sigma^{-}\right)=\alpha^{\prime} p_{R}^{-} \sigma^{-}$, with $\sqrt{2} p_{L}^{+}=\sqrt{2} p_{R}^{-}=p_{o}^{0}$.
} 
Here and in the following, we show explicitly the dependence of the closed-open vertex on the transverse momentum carried by the closed state on which it acts. We will use this choice of frame to check that the closed-open vertex correctly reproduces the imaginary part of the elastic scattering of the tachyon for the first massive level of the spectrum in appendix A. In the Regge limit it is sometimes more convenient to choose

$$
p_{1}=\left(E, \overrightarrow{0}_{p}, \vec{q}, \sqrt{p^{2}-\vec{q}^{2}}\right), \quad p_{2}=\left(-E, \overrightarrow{0}_{p}, \overrightarrow{0}_{24-p},-p\right) .
$$

In this frame the relation $t=-\left(p_{1}+p_{2}\right)^{2} \sim-\vec{q}^{2}$ remains valid up to terms of order $s^{-1}$, which are negligible in the high energy limit if we are only interested in the leading order.

\section{The high-energy limit of the closed-open vertex}

In the previous section we summarized the form of the vertex that gives the transition amplitude between an arbitrary closed string state and an arbitrary open string state. We now discuss how the structure of this operator simplifies when the absorbed string is ultrarelativistic, $\alpha^{\prime} s \gg 1$ and $M \ll E$. In this section and in the rest of the paper we will work in the light-cone gauge parallel to the collision axis, or more precisely to the direction of large momentum, since it is in this gauge that we will be able to give a very explicit description of the massive open state created on the brane worldvolume. As a check of our construction we will verify that starting from the high-energy limit of the closed-open vertex we can reproduce the imaginary part of the elastic amplitude, which is due to the creation of on-shell states in the $s$-channel. This analysis complements the analysis performed in [5] where we studied the Regge limit of the three closed-string lightcone vertex, which gives all the inelastic closed-closed transitions at high energy in the background of the $\mathrm{D} p$-branes.

Consider a closed state $|\psi\rangle$ at level $N_{c}$, with energy $E$ such that

$$
\alpha^{\prime} E^{2}=n-1
$$

and carrying a momentum $\vec{p}_{t}$ in the transverse directions, $p_{t}^{2} \ll E^{2}$. The non-trivial part of the closed-open vertex in eq. (3.9) when acting on this state can be written as

$$
V_{\vec{p}}|\psi\rangle=\beta \mathcal{P}_{n} e^{Z_{o}+Z_{c, \psi}}|\psi\rangle,
$$

where $Z_{o}$ contains only the open string modes (i.e. the Neumann coefficients $N_{k l}^{33}$ and $N_{k}^{3}$ ) and $Z_{c, \psi}$ contains all the remaining terms which have both open and closed string modes, the latter restricted to those that can have a non-vanishing contraction with the modes used to define the closed state The operator $\mathcal{P}_{n}$ is the projector on the level $n$ of the open string Hilbert space and enforces energy conservation. We shall derive the asymptotic behaviour of the vertex (4.2) in the large $n$ limit

$$
V_{\vec{p}_{t}} \sim \mathcal{V}_{\vec{p}_{t}}, \quad n \gg 1 \text {. }
$$

The asymptotic vertex gives the couplings between a highly energetic closed string $|\psi\rangle$ and a very massive open string $|\chi\rangle$

$$
\mathcal{B}_{\psi \chi}=\left\langle\chi\left|\mathcal{V}_{\vec{p}_{t}}\right| \psi\right\rangle
$$


The imaginary part of the elastic disk amplitude in the Regge limit can be computed combining two closed-open vertices

$$
\operatorname{Im} \mathcal{A}_{\psi \psi}(s, t)=\pi \alpha^{\prime}\left\langle\psi\left|\mathcal{V}_{\overrightarrow{0}}^{\dagger} \mathcal{V}_{\vec{q}}\right| \psi\right\rangle, \quad \alpha^{\prime} s=n-1, \quad t=-\vec{q}^{2} .
$$

We will also be interested in the closed-open couplings and in the imaginary part of the elastic disk amplitude in impact parameter space

$$
\begin{gathered}
\mathcal{B}_{\psi \chi}(\vec{b})=\int \frac{d^{24-p} q}{(2 \pi)^{24-p}} e^{-i \vec{b} \vec{q}}\left\langle\chi\left|\mathcal{V}_{\vec{q}}\right| \psi\right\rangle \equiv\left\langle\chi\left|\widetilde{\mathcal{V}}_{\vec{b}}\right| \psi\right\rangle, \\
\operatorname{Im} \mathcal{A}_{\psi \psi}(s, \vec{b})=\pi \alpha^{\prime} \int \frac{d^{24-p} q}{(2 \pi)^{24-p}} e^{-i \vec{b} \vec{q}}\left\langle\psi\left|\mathcal{V}_{0}^{\dagger} \mathcal{V}_{\vec{q}}\right| \psi\right\rangle \equiv \pi \alpha^{\prime}\left\langle\psi\left|\mathcal{V}_{0}^{\dagger} \widetilde{\mathcal{V}}_{\vec{b}}\right| \psi\right\rangle,
\end{gathered}
$$

where we introduced the vertex in impact parameter space

$$
\widetilde{\mathcal{V}}_{\vec{b}}=\int \frac{d^{24-p} q}{(2 \pi)^{24-p}} e^{-i \vec{b} \vec{q}} \mathcal{V}_{\vec{q}}
$$

As we shall see, at high energy the Fourier transforms in the previous expressions are dominated by the region of small transverse momenta and therefore we will find a simple relation between the vertex in impact parameter space and the vertex at zero transverse momentum.

In order to derive the high energy limit of the vertex, we must determine the asymptotic behaviour of the Neumann coefficients. At high energy the ratios of the light-cone components $p^{+}$of the momenta of the three strings that appear in eq. (3.16) behave as follows:

$$
\begin{aligned}
& \frac{\alpha_{3}}{\alpha_{2}}=-\frac{2 m}{E-p} \sim-\frac{n}{\omega}, \\
& \frac{\alpha_{2}}{\alpha_{1}}=\frac{E-p}{E+p} \sim \frac{\omega}{n}, \\
& \frac{\alpha_{1}}{\alpha_{3}}=-\frac{E+p}{2 m} \sim-1+\frac{\omega}{n},
\end{aligned}
$$

where the $\alpha_{i}$ are defined in eq. (3.20) and $\omega$ is defined in eq. (3.21). Using these expressions we can analyze the high energy limit of the Neumann coefficients $N_{k l}^{r s}$ and $N_{k}^{r} \vec{P}$. When $n$ tends to infinity, the coefficients quadratic in the open modes behave like

$$
N_{k l}^{33} \sim-\frac{\omega}{n} \frac{1}{k+l} \frac{k^{-\omega \frac{k}{n}}}{\Gamma\left(1-\omega \frac{k}{n}\right)} \frac{l^{-\omega \frac{l}{n}}}{\Gamma\left(1-\omega \frac{l}{n}\right)} .
$$

If in the large $n$ limit the ratios $k / n$ and $l / n$ tend to zero this is

$$
N_{k l}^{33} \sim-\frac{\omega}{n} \frac{1}{k+l} .
$$

On the other hand, if the open modes are of order $n$ setting

$$
k=n x, \quad l=n y,
$$


we find

$$
N_{k l}^{33} \sim-n^{-\omega(x+y)-2} \frac{x^{-\omega x} y^{-\omega y}}{\Gamma(1-\omega x) \Gamma(1-\omega y)} \frac{\omega}{x+y} .
$$

The coefficients quadratic in the closed modes have the following behaviour:

$$
\begin{aligned}
& N_{k l}^{11} \sim \frac{\omega}{n} \frac{(-1)^{k+l}}{k+l}, \\
& N_{k l}^{22} \sim\left(\frac{n}{\omega}\right)^{k+l} \frac{k^{k}}{k !} \frac{l^{l}}{l !} \frac{1}{k+l}, \\
& N_{k l}^{12} \sim(-1)^{k+1}\left(\frac{n}{\omega}\right)^{l-1} \frac{l^{l-1}}{l !} .
\end{aligned}
$$

The left and right closed string modes $k, l$ are always much smaller than $n$. Note that the coefficients $N_{k l}^{22}$ scale like a positive power of $n$. The mixed coefficients with one closed and one open mode behave generically like

$$
\begin{aligned}
& N_{k l}^{13} \sim \frac{(-1)^{k}}{l-k} \frac{\omega}{n} \frac{l^{-\omega \frac{l}{n}}}{\Gamma\left(1-\omega \frac{l}{n}\right)}, \\
& N_{k l}^{23} \sim \frac{1}{k-\omega \frac{l}{n}} \frac{k^{k}}{k !}\left(\frac{n}{\omega}\right)^{k-1} \frac{l^{-\omega \frac{l}{n}}}{\Gamma\left(1-\omega \frac{l}{n}\right)} .
\end{aligned}
$$

The coefficients $N_{k l}^{13}$ possess an interesting feature. They are enhanced by an additional power of $n$ when the open and the closed indices coincide

$$
N_{k k}^{13} \sim-\frac{(-1)^{k}}{k} \frac{n}{\omega k} \frac{\sin \left(\pi \omega \frac{k}{n}\right)}{\pi} \sim-\frac{(-1)^{k}}{k},
$$

where the last approximation holds since $k \ll n$. This feature will lead to a simple formula for the absorption of a generic closed string state. Finally the coefficients linear in the string modes behave as follows:

$$
\begin{aligned}
& N_{k}^{1} \vec{P} \sim \frac{(-1)^{k}}{k} \sqrt{\frac{\alpha^{\prime}}{2}} \vec{p}_{t}, \\
& N_{k}^{2} \vec{P} \sim-\frac{k^{k}}{k !}\left(\frac{n}{\omega}\right)^{k} \frac{1}{k} \sqrt{\frac{\alpha^{\prime}}{2}} \vec{p}_{t}, \\
& N_{k}^{3} \vec{P} \sim \frac{k^{-\omega \frac{k}{n}}}{\Gamma\left(1-\omega \frac{k}{n}\right)} \frac{1}{k} \sqrt{\frac{\alpha^{\prime}}{2}} \vec{p}_{t} .
\end{aligned}
$$

\section{Closed-open transitions at high energy}

We are now ready to derive the explicit form of the highly excited open state created in the absorption process of a very energetic closed string. For clarity, we will analyze separately the absorption of a closed string tachyon, of a massless closed string state and of a state belonging to the first massive level of the closed string spectrum. We will then determine the open state created by the absorption of an arbitrary closed state.

We will start by considering the case in which the closed state carries zero transverse momentum, showing how to reconstruct the discontinuity of the elastic amplitude at $t=0$. 
We will then include a transverse momentum $\vec{p}_{t}$ (with $p_{t}^{2} \ll E^{2}$ ) and derive the form of the open state created at fixed impact parameter $b$. Finally, we will check that this very massive open string state reproduces the imaginary part of the elastic closed string amplitude in the Regge limit and for arbitrary values of $b$.

\subsection{Tachyon}

We begin for simplicity with the vertex that describes the transition of a closed string tachyon with $\vec{p}_{t}=0$ to an open string state with mass $\alpha^{\prime} m^{2}=n-1$

$$
\mathcal{V}_{\overrightarrow{0}}|0\rangle=\beta \mathcal{P}_{n} e^{\frac{1}{2} \sum_{k, l} N_{k l}^{33} a_{-k}^{i} a_{-l}^{i}|0\rangle,}
$$

where for large $n$

$$
N_{k l}^{33} \sim n^{x+y-2} \frac{x^{x} y^{y}}{\Gamma(1+x) \Gamma(1+y)} \frac{1}{x+y}, \quad k=n x, \quad l=n y,
$$

which follows from eq. (4.13) with $\omega=-1$. Expanding the exponential in eq. (5.1), we obtain the following series representations for the open state:

$$
\mathcal{V}_{\overrightarrow{0}}|0\rangle=\beta \sum_{Q=0}^{\infty} \frac{1}{Q ! 2^{Q}} \mathcal{P}_{n} \prod_{\alpha=1}^{Q} \sum_{k_{\alpha}, l_{\alpha}} N_{k_{\alpha} l_{\alpha}}^{33} a_{-k_{\alpha}}^{i_{\alpha}} a_{-l_{\alpha}}^{i_{\alpha}}|0\rangle,
$$

and for the imaginary part of the elastic amplitude

$$
\operatorname{Im} \mathcal{A}_{T T}=\pi \alpha^{\prime} \beta^{2} \sum_{Q=0}^{\infty} \frac{1}{(Q !)^{2} 2^{2 Q}} \sum_{\left\{k_{\alpha}, l_{\alpha}\right\}} \prod_{\alpha=1}^{Q}\left(N_{k_{\alpha} l_{\alpha}}^{33}\right)^{2}\left\langle 0\left|\prod_{\alpha=1}^{Q} a_{k_{\alpha}}^{i_{\alpha}} a_{l_{\alpha}}^{i_{\alpha}} \prod_{\alpha=1}^{Q} a_{-k_{\alpha}}^{i_{\alpha}} a_{-l_{\alpha}}^{i_{\alpha}}\right| 0\right\rangle,
$$

where the integers $k_{\alpha}, l_{\alpha}$ satisfy the constraint $\sum_{\alpha=1}^{Q}\left(k_{\alpha}+l_{\alpha}\right)=n$. It is remarkable that at high energy and in the gauge aligned to the large momentum, it is sufficient to keep only the first few terms in the previous series in order to get a very accurate representation of the state.

Let us evaluate the first term in the series in eq. (5.4), which approximates the open state with the state created by the action of just one couple of open modes $a_{-k}^{i} a_{-l}^{i}$

$$
\operatorname{Im} \mathcal{A}_{T T} \sim \pi \alpha^{\prime} \beta^{2} \sum_{\substack{k, l \\ k+l=n}} \frac{1}{4}\left(N_{k l}^{33}\right)^{2}\left\langle 0\left|a_{k}^{i} a_{l}^{i} a_{-k}^{j} a_{-l}^{j}\right| 0\right\rangle=\pi \alpha^{\prime} \beta^{2} \sum_{\substack{k, l \\ k+l=n}} 12\left(N_{k l}^{33}\right)^{2} k l,
$$

where we used the fact that the indices $i, j$ run from 1 to 24 . At high energy we can evaluate the previous sum by approximating it with an integral, using eq. (5.2) and the fact that, for large $n, \sum_{k, l} \rightarrow n^{2} \int_{0}^{1} d x \int_{0}^{1} d y$ and $\delta_{k+l, n} \rightarrow \frac{1}{n} \delta(x+y-1)$

$$
\operatorname{Im} \mathcal{A}_{T T} \sim 12 \pi \alpha^{\prime} \beta^{2} n \int_{0}^{1} d x \int_{0}^{1} d y \delta(x+y-1) \frac{x^{2 x+1} y^{2 y+1}}{\Gamma^{2}(1+x) \Gamma^{2}(1+y)} .
$$

Evaluating the integral we find that this approximation to the complete open state already accounts for 0.93 of the total imaginary part

$$
\operatorname{Im} \mathcal{A}_{T T} \sim 0.929 \pi \alpha^{\prime} \beta^{2} n
$$


Note that since in this case the product of the Neumann coefficients $\left(N_{k l}^{33}\right)^{2} k l$ give a contribution of order one, the power of $n$ required by the discontinuity of the elastic amplitude is entirely due to the multiplicity of the possible states (the number of partitions of $n$ into two integers). In general for any given term of the series in eq. (5.4) containing $Q$ couples of oscillators the overall power of $n$ results from a power of $n^{2-2 Q}$ from the product of the Neumann coefficients and a power of $n^{2 Q-1}$ from the sum over all possible states.

Consider for instance the second term in the series. There are now eight modes in the matrix element and two classes of inequivalent contractions among them, giving respectively a double trace and a single trace in the transverse indices. In the first case we have

$$
72 \int d x_{1} d y_{1} d x_{2} d y_{2} \prod_{i=1}^{2} \frac{x_{i}^{2 x_{i}+1} y_{i}^{2 y_{i}+1}}{\Gamma^{2}\left(x_{i}+1\right) \Gamma^{2}\left(y_{i}+1\right)} \frac{\delta\left(x_{1}+y_{1}+x_{2}+y_{2}-1\right)}{\left(x_{1}+y_{1}\right)^{2}\left(x_{2}+y_{2}\right)^{2}}
$$

and in the second

$$
6 \int d x_{1} d y_{1} d x_{2} d y_{2} \prod_{i=1}^{2} \frac{x_{i}^{2 x_{i}+1} y_{i}^{2 y_{i}+1}}{\Gamma^{2}\left(x_{i}+1\right) \Gamma^{2}\left(y_{i}+1\right)} \frac{\delta\left(x_{1}+y_{1}+x_{2}+y_{2}-1\right)}{\left(x_{1}+y_{1}\right)\left(y_{1}+x_{2}\right)\left(x_{2}+y_{2}\right)\left(y_{2}+x_{1}\right)},
$$

where in both cases we omitted a factor $\pi \alpha^{\prime} \beta^{2} n$. Adding the contribution of these two terms to the leading contribution we obtain

$$
\operatorname{Im} \mathcal{A}_{T T} \sim 0.998 \pi \alpha^{\prime} \beta^{2} n
$$

Therefore only $0.2 \%$ of the full forward imaginary part is left to terms with six or more oscillators. This is strong evidence that the series converges rapidly and that

$$
\left\langle 0\left|e^{Z_{0}^{\dagger}} \mathcal{P}_{n} e^{Z_{0}}\right| 0\right\rangle=n
$$

as required by eq. (4.5).

Let us now consider the absorption amplitude and the imaginary part of the disk at fixed impact parameter. We will find that the open state is closely related to the one created by the tachyon when $\vec{p}_{t}=0$. We start from the vertex with a non-vanishing transverse momentum $\vec{q}$ and evaluate its Fourier transform. For clarity, in the following we will display explicitly the dependence of the Neumann coefficients on the transverse momentum $\vec{q}$, writing $N_{k l}^{r s}(\vec{q})$ and $N_{k}^{r}(\vec{q})$.

When the transverse momentum is non zero, the vertex becomes

$$
\mathcal{V}_{\vec{q}}|0\rangle=\beta \mathcal{P}_{n} e^{\frac{1}{2} \sum_{k, l} N_{k l}^{33}(\vec{q}) a_{-k}^{i} a_{-l}^{i}+\sum_{k} N_{k}^{3}(\vec{q}) P^{i} a_{-k}^{i}|0\rangle,}
$$

where

$$
\begin{aligned}
N_{k l}^{33}(\vec{q}) & \sim \frac{k^{(1-\lambda) \frac{k}{n}} l^{(1-\lambda) \frac{l}{n}}}{\Gamma\left(1+(1-\lambda) \frac{k}{n}\right) \Gamma\left(1+(1-\lambda) \frac{l}{n}\right)} \frac{(1-\lambda)}{n(k+l)}, \quad \lambda=\frac{\alpha^{\prime} \vec{q}^{2}}{4}, \\
N_{k}^{3}(\vec{q}) \vec{P} & \sim \frac{k^{(1-\lambda) \frac{k}{n}}}{\Gamma\left(1+(1-\lambda) \frac{k}{n}\right)} \frac{1}{k} \sqrt{\frac{\alpha^{\prime}}{2}} \vec{q} .
\end{aligned}
$$


Expanding the exponential in eq. (5.12), it is easy to see that the linear and the quadratic Neumann coefficients give contributions of the same order in the energy. It is however possible to find a very simple form for the closed-open vertex in impact parameter space. The main observation is that the essential dependence of the coefficients $N_{k l}^{33}(\vec{q})$ and $N_{k}^{3}(\vec{q})$ on the transverse momentum is in the factors $k^{-\lambda \frac{k}{n}}$ or, with $k=n x$,

$$
(n x)^{-\lambda x}=e^{-\frac{\alpha^{\prime} \vec{q}^{2}}{4} x \log (n x)} \sim e^{-\frac{\alpha^{\prime} \vec{q}^{2}}{4} x \log n},
$$

since $x \log x \ll x \log n$. In order to evaluate

$$
\widetilde{\mathcal{V}}_{\vec{b}}|0\rangle=\int \frac{d^{24-p} q}{(2 \pi)^{24-p}} e^{-i \vec{b} \vec{q}} \mathcal{V}_{\vec{q}}|0\rangle
$$

we expand the Neumann coefficients in eq. (5.13) in a power series in $\vec{q}^{2}$, retaining however in each term the $\vec{q}^{2}$-dependent powers of the energy in eq. (5.14). We then expand the exponential rewriting the operator in eq. (5.12) as follows:

$$
\mathcal{V}_{\vec{q}}=\mathcal{W}\left[1+\vec{q}^{2} O_{2}+\vec{q}^{2} q^{i} O_{3}^{i}+\ldots+q^{i_{1}} \ldots q^{i_{k}} O_{k}^{i_{1} \ldots i_{k}}+\ldots\right],
$$

where the operator $\mathcal{W}$ is given by

$$
\mathcal{W}=\beta \mathcal{P}_{n} e^{\frac{1}{2} \sum_{k, l} W_{k l}^{33} a_{-k}^{i} a_{-l}^{i}+\sqrt{\frac{\alpha^{\prime}}{2}} \sum_{k} W_{k}^{3} a_{-k}^{i} q^{i}}|0\rangle,
$$

with

$$
W_{k l}^{33}=N_{k l}^{33}(0) e^{-\frac{\alpha^{\prime} \vec{q}^{2}}{4} \frac{k+l}{n} \log n}, \quad W_{k}^{3}=\frac{k^{\frac{k}{n}-1}}{\Gamma\left(1+\frac{k}{n}\right)} e^{-\frac{\alpha^{\prime} \vec{q}^{2}}{4} \frac{k}{n} \log n} .
$$

Here $N_{k l}^{33}(0)$ are the Neumann coefficients at zero transverse momentum in (5.1) and the coefficients $W_{k l}^{33}$ coincide with them except for the Gaussian factor in $\vec{q}$. Note also that we kept in the exponential the terms linear in $\vec{q}$ and in the open modes.

We can evaluate the contribution of the first term in the series following the same steps as at the beginning of this section. We find

$$
\begin{aligned}
\widetilde{\mathcal{V}}_{\vec{b}}|0\rangle & \sim \beta \int \frac{d^{24-p} q}{(2 \pi)^{24-p}} e^{-i \vec{b} \vec{q}} \mathcal{P}_{n}\left[e^{\frac{1}{2} \sum_{k, l} W_{k l}^{33} a_{-k}^{i} a_{-l}^{i}+\sqrt{\frac{\alpha^{\prime}}{2}} \sum_{k} W_{k}^{3} a_{-k}^{i} q^{i}}\right]|0\rangle \\
& =\beta \int \frac{d^{24-p} q}{(2 \pi)^{24-p}} e^{-i \vec{b} \vec{q}} e^{-\frac{\alpha^{\prime} \vec{q}^{2}}{4} \log n} \mathcal{P}_{n}\left[e^{\frac{1}{2} \sum_{k, l} N_{k l}^{33}(0) a_{-k}^{i} a_{-l}^{i}+\sqrt{\frac{\alpha^{\prime}}{2}} \sum_{k} \frac{k^{\frac{k}{n}}-1}{\Gamma\left(1+\frac{k}{n}\right)} a_{-k}^{i} q^{i}}\right]|0\rangle \\
& =\frac{1}{\left(\pi \alpha^{\prime} \log \alpha^{\prime} s\right)^{\frac{24-p}{2}}} \mathcal{P}_{n}\left[e^{-\frac{1}{\alpha^{\prime} \log \alpha^{\prime} s}\left(b^{i}+i \sqrt{\frac{\alpha^{\prime}}{2}} \sum_{k} \frac{1}{k} a_{-k}^{i}\right)^{2}} \mathcal{V}_{\overrightarrow{0}}\right]|0\rangle
\end{aligned}
$$

In the second line we used that, due to the presence of the projector, the $\vec{q}^{2}$-dependent powers of the energy in the coefficients $W_{k_{\alpha} l_{\alpha}}^{33}$ and $W_{k_{\alpha}}^{3}$ combine in an overall Gaussian factor. In the last line we approximated $k / n \sim 0$ in the operator multiplying $\mathcal{V}_{\overrightarrow{0}}$ since in impact parameter space the leading contributions due to this operator arise from modes with $k \ll n$. 
The Fourier transform of the first term in the series in eq. (5.16) thus coincides with the vertex at zero transverse momentum up to the insertion of an operator with an overall dependence on $b$ given by a Gaussian form factor

$$
e^{-\frac{b^{2}}{\alpha^{\prime} \log \alpha^{\prime} s}}
$$

Note that the length scale in this form factor is the string scale enhanced by a logarithm of the energy

$$
\alpha^{\prime} \log \alpha^{\prime} s .
$$

This is due to the well-known logarithmic growth of a highly energetic string in the transverse directions. Precisely as a consequence of this phenomenon, all the other contributions in the series in eq. (5.16) are subleading in the high-energy limit and the term we have just evaluated gives the full answer.

To verify this we do not need the precise form of the operator coefficients $O^{i_{1} \ldots i_{k}}$, all that is relevant is that each term is multiplied by the Gaussian factor in eq. (5.20). When we evaluate the Fourier transform of the other terms in the series, the powers of the transverse momentum become derivatives with respect to the impact parameter and the resulting contribution is then suppressed by inverse powers of $\log \alpha^{\prime} s$. The final result is therefore

$$
\tilde{\mathcal{V}}_{\vec{b}}|0\rangle=\frac{1}{\left(\pi \alpha^{\prime} \log \alpha^{\prime} s\right)^{\frac{24-p}{2}}} \mathcal{P}_{n}\left[e^{-\frac{1}{\alpha^{\prime} \log \alpha^{\prime} s}\left(b^{i}+i \sqrt{\frac{\alpha^{\prime}}{2}} \sum_{k} \frac{1}{k} a_{-k}^{i}\right)^{2}} \mathcal{V}_{\overrightarrow{0}}\right]|0\rangle .
$$

This is the form of the vertex that we will use in actual calculations. It can also be written in a very suggestive form if we interpret the Gaussian factor as a squeezed state for the effective creation and destruction operators

$$
B^{i}=\frac{1}{\sqrt{\log n}} \sum_{k=1}^{n} \frac{a_{k}^{i}}{k}, \quad B^{i \dagger}=\frac{1}{\sqrt{\log n}} \sum_{k=1}^{n} \frac{a_{-k}^{i}}{k} .
$$

In the high energy limit they satisfy

$$
\left[B^{i}, B^{j \dagger}\right]=\frac{\delta_{i j}}{\log n} \sum_{k=1}^{n} \frac{1}{k} \sim \delta_{i j}
$$

Since the squeezed states represent the position eigenstates in the oscillator basis of the Hilbert space we can write

$$
|b\rangle_{X}=\frac{1}{\left(\sqrt{\pi} l_{n}\right)^{\frac{24-p}{2}}} e^{-\frac{b^{2}}{2 l_{n}^{2}}-i \frac{\sqrt{2}}{l_{n}} B^{\dagger} b+\frac{1}{2}\left(B^{\dagger}\right)^{2}}|0\rangle=e^{-i b \vec{P}}|0\rangle_{X}, \quad l_{n}^{2}=\alpha^{\prime} \log n,
$$

where $|b\rangle_{X}$ is an eigenstate of the position operators

$$
X^{i}=i \frac{l_{n}}{\sqrt{2}}\left(B^{i}-B^{i \dagger}\right)=i \sqrt{\frac{\alpha^{\prime}}{2}} \sum_{k=1}^{n}\left(\frac{a_{k}^{i}}{k}-\frac{a_{-k}^{i}}{k}\right) \equiv \sum_{k=1}^{n} x_{k}^{i},
$$


with eigenvalue $b^{i}$ and the $P^{i}$ are the corresponding momentum operators

$$
P^{i}=\frac{1}{\sqrt{2} l_{n}}\left(B^{i}+B^{i \dagger}\right)=\frac{1}{\sqrt{2 \alpha^{\prime}} \log \alpha^{\prime} s} \sum_{k=1}^{n}\left(\frac{a_{k}^{i}}{k}+\frac{a_{-k}^{i}}{k}\right) \equiv \frac{1}{\log \alpha^{\prime} s} \sum_{k=1}^{n} p_{k}^{i} .
$$

Here $x_{k}$ and $p_{k}$ are the position and momentum modes of the open string, as defined in the two previous equations. The state $|b\rangle_{X}$ is normalized in the standard way, $\left\langle b \mid b^{\prime}\right\rangle=\delta\left(b-b^{\prime}\right)$. We can then write

$$
\widetilde{\mathcal{V}}_{\vec{b}}|0\rangle=\frac{e^{-\frac{b^{2}}{2 \alpha^{\prime} \log \alpha^{\prime} s}}}{\left(\pi \alpha^{\prime} \log \alpha^{\prime} s\right)^{\frac{24-p}{4}}} \mathcal{P}_{n} \mathcal{V}_{\overrightarrow{0}}|b\rangle_{X}
$$

This form of the closed-open vertex in impact parameter space is very natural. The appearance of the squeezed state $|b\rangle_{X}$ indicates that the closed-open transition is proportional to a delta function $\delta(X-b)$ for the effective position operator $X$. Its presence reflects the locality of the string interactions, the light-cone vertex allowing only transitions between closed and open strings that overlap along their entire length. This requires in particular that the closed string should touch the branes in at least one point in order for the transition to take place. The way that a closed string initially localized away from the location of the branes manages to touch them and split is via quantum fluctuations in its position.

From eq. (5.22) we see in fact that the transition happens with a non negligible amplitude as long as $b^{2} \leq \alpha^{\prime} \log \alpha^{\prime} s$, that is for impact parameters that can be much larger than the maximal size $\alpha^{\prime} M$ of a string of mass $M$, which is kept fixed in the high energy limit. We can picture the transition as happening in two stages. First the closed string becomes polarized, stretching towards the branes so that it touches them in at least one point, then it splits to form the final open string. The Gaussian dependence on the impact parameter of the transition amplitude can then be related to the probability amplitude of finding the closed string in a configuration where it overlaps with the branes. We will confirm this picture in [14] where we will discuss the classical open string solutions that correspond to the state created on the branes in the closed-open transition.

Let us finally note that using eq. (5.28) one can prove that states created at different values of the impact parameter are orthogonal. This follows from the fact that at high energy $\mathcal{P}_{n} \mathcal{V}_{0}|b\rangle_{X}$ remains to a first approximation an eigenstate of the transverse position opertors $X^{i}$.

Using this vertex we can evaluate the discontinuity of the elastic amplitude in impact parameter space. The calculation is most easily done representing the imaginary part as the product of the vertex at impact parameter $b$ and the vertex at zero momentum, as in eq. (4.7)

$$
\operatorname{Im} \mathcal{A}_{T T}(s, b)=\pi \alpha^{\prime}\left\langle 0\left|\mathcal{V}_{\overrightarrow{0}}^{\dagger} \widetilde{\mathcal{V}}_{\vec{b}}\right| 0\right\rangle
$$

In order to evaluate

$$
\left\langle 0\left|\mathcal{V}_{\overrightarrow{0}}^{\dagger} \mathcal{P}_{n}\left[e^{-\frac{1}{\alpha^{\prime} \log \alpha^{\prime} s}\left(b^{i}+i \sqrt{\frac{\alpha^{\prime}}{2}} \sum_{k} \frac{1}{k} a_{-k}^{i}\right)^{2}} \mathcal{V}_{\overrightarrow{0}}\right]\right| 0\right\rangle
$$


it is sufficient to notice that, compared with the calculation done for $t=0$, the additional terms in the previous formula give contributions that are suppressed by powers of $\log \alpha^{\prime} s$. We then find

$$
\operatorname{Im} \mathcal{A}_{T T}(s, b)=\pi \alpha^{\prime} \beta^{2} \alpha^{\prime} s \frac{e^{-\frac{b^{2}}{\alpha^{\prime} \log \alpha^{\prime} s}}}{\left(\pi \alpha^{\prime} \log \alpha^{\prime} s\right)^{\frac{24-p}{2}}},
$$

reproducing eq. (2.10). Here the factor $\alpha^{\prime} s \equiv n$ comes from the matrix element containing two vertices at $t=0$, as we have already seen in eqs. (5.6), (5.10) and (5.11).

\subsection{Massless states}

We now discuss the absorption of a massless closed string state

$$
\left|g_{\epsilon}, \bar{g}_{\bar{\epsilon}}\right\rangle=\left(\epsilon A_{-1}\right)\left(\bar{\epsilon} \bar{A}_{-1}\right)|0\rangle,
$$

with polarization tensor $\epsilon_{i} \bar{\epsilon}_{j}$. The open state created by the absorption of a very energetic massless closed string has an extremely simple description, simpler than the one we found for the absorption of a tachyon. When the transverse momentum vanishes it is given by

$$
\mathcal{V}_{\overrightarrow{0}}\left|g_{\epsilon}, \bar{g}_{\bar{\epsilon}}\right\rangle=\beta\left(\epsilon a_{-1}\right)\left(a_{-n+1} D \bar{\epsilon}\right)|0\rangle .
$$

In order to derive this result we have to deal with some technical complications, which arise because our light-cone gauge is not very suitable to describe the right part of a closed massless state with zero transverse momentum. The action of the reflection matrix $D^{\mu \nu}$ makes it equivalent to a state with $p^{+}=0$, see $\alpha_{2}$ in (3.20) with $N_{c}=1$ and $\vec{p}_{t}=0$. We could simply evaluate the transition amplitudes with $\vec{p}_{t} \neq 0$ and then take the limit $\vec{p}_{t} \rightarrow 0$. However it turns out that in order to have a well defined limit it is necessary to give a small mass $\mu$ to the massless state and send first $\vec{p}_{t}$ to zero and then $\mu$ to zero. Otherwise the result of the limit would depend on the direction of $\vec{p}_{t}$, as a consequence of the singular behaviour of the Neumann coefficients $N_{k}^{2}$. A more detailed discussion of this subtlety can be found in appendix A, where we make a direct comparison between a covariant amplitude and an amplitude derived using the light-cone vertex and show that the two agree when the limit of zero transverse momentum is taken as explained above.

When acting on a massless state the general closed-open vertex becomes

$$
V_{\vec{p}_{t}}\left|g_{\epsilon}, \bar{g}_{\bar{\epsilon}}\right\rangle=\beta \mathcal{P}_{n} e^{Z_{o}+Z_{c, g}}\left|g_{\epsilon}, \bar{g}_{\bar{\epsilon}}\right\rangle
$$

where

$$
\begin{aligned}
Z_{c, g} & =N_{11}^{12} A_{1} D \bar{A}_{1}+N_{1 u}^{23} \bar{A}_{1} D a_{-u}+N_{1 u}^{13} A_{1} a_{-u}+N_{1}^{1} \vec{P} A_{1}+N_{1}^{2} \vec{P} D \bar{A}_{1}, \\
Z_{o} & =\frac{1}{2} N_{k l}^{33} a_{-k} a_{-l}+N_{u}^{3} P a_{-u} .
\end{aligned}
$$

Therefore

$$
\begin{aligned}
V_{\overrightarrow{p_{t}}}\left|g_{\epsilon}, \bar{g}_{\bar{\epsilon}}\right\rangle= & \beta \mathcal{P}_{n} e^{Z_{o}}\left[(\epsilon D \bar{\epsilon}) N_{11}^{12}+N_{1 u}^{23} N_{1 v}^{13}\left(\epsilon a_{-v}\right)\left(a_{-u} D \bar{\epsilon}\right)+N_{1 u}^{23} N_{1}^{1}\left(a_{-u} D \bar{\epsilon}\right)(\epsilon \vec{P})\right. \\
& \left.+N_{1 u}^{13} N_{1}^{2}\left(\epsilon a_{-u}\right)(\bar{\epsilon} D \vec{P})+N_{1}^{1} N_{1}^{2}(\bar{\epsilon} D \vec{P})(\epsilon \vec{P})\right]|0\rangle .
\end{aligned}
$$


This expression simplifies considerably in the high-energy limit. Let us set

$$
\lambda=\frac{\alpha^{\prime}}{4} \vec{p}_{t}^{2}+\alpha^{\prime} \mu^{2}
$$

where $\mu$ is the small mass introduced to regularize the limit $\vec{p}_{t} \rightarrow 0$. For large $n$ the Neumann coefficients in eq. (5.36) behave as follows:

$$
N_{11}^{12} \sim 1, \quad N_{1 k}^{13} \sim-\frac{1}{k-1} \frac{\lambda}{n} \frac{k^{-\lambda \frac{k}{n}}}{\Gamma\left(1-\lambda \frac{k}{n}\right)}, \quad k \neq 1, \quad N_{11}^{13} \sim 1 .
$$

Note the enhancement of the contractions between left modes and open modes when the mode numbers coincide. We also have

$$
N_{1 k}^{23} \sim \frac{n}{n-\lambda k} \frac{k^{-\lambda \frac{k}{n}}}{\Gamma\left(1-\lambda \frac{k}{n}\right)}, \quad N_{k l}^{33} \sim-\frac{\lambda}{n(k+l)} \frac{k^{-\lambda \frac{k}{n}}}{\Gamma\left(1-\lambda \frac{k}{n}\right)} \frac{l^{-\lambda \frac{l}{n}}}{\Gamma\left(1-\lambda \frac{l}{n}\right)},
$$

and

$$
N_{1}^{1} \vec{P} \sim-\sqrt{\frac{\alpha^{\prime}}{2}} \vec{p}_{t}, \quad N_{1}^{2} \vec{P} \sim-\frac{n}{\lambda} \sqrt{\frac{\alpha^{\prime}}{2}} \vec{p}_{t}, \quad N_{k}^{3} \vec{P} \sim \frac{1}{k} \frac{k^{-\lambda \frac{k}{n}}}{\Gamma\left(1-\lambda \frac{k}{n}\right)} \sqrt{\frac{\alpha^{\prime}}{2}} \vec{p}_{t} .
$$

Focusing on the energy dependence of the Neumann coefficients, we can see that the fourth term in eq. (5.36) would give a leading contribution of order $n$ which however disappears in the $\vec{p}_{t} \rightarrow 0$ limit. Similarly we can discard all other terms containing factors of $\vec{P}$ which vanish in the $\vec{p}_{t} \rightarrow 0$ limit thanks to the presence of the mass $\mu$. Thus the next most relevant term is the second when $v=1$ and $u=n-1$, so as to use the last in (5.38), and is of order $\sqrt{n}$ when written in terms of mode operators that commute to one. Therefore the leading terms at high energy are

$$
\mathcal{V}_{\vec{p}_{t}}\left|g_{\epsilon}, \bar{g}_{\bar{\epsilon}}\right\rangle \sim \beta \mathcal{P}_{n}\left[e^{Z_{o}} N_{11}^{13} N_{1 u}^{23}\left(\epsilon a_{-1}\right)\left(a_{-u} D \bar{\epsilon}\right)\right]|0\rangle .
$$

Let us now take the limit $\vec{p}_{t} \rightarrow 0$ on $Z_{o}$. Since $N_{k l}^{33}$ vanish when we set $\mu=0$ we also have $e^{Z_{o}} \sim 1$. Taking into account the projector $\mathcal{P}_{n}$ and using that $N_{1, n-1}^{23} \sim n^{-\alpha^{\prime} \mu^{2}}$ we find the state in eq. (5.33)

$$
\mathcal{V}_{\overrightarrow{0}}\left|g_{\epsilon}, \bar{g}_{\bar{\epsilon}}\right\rangle=\beta\left(\epsilon a_{-1}\right)\left(a_{-n+1} D \bar{\epsilon}\right)|0\rangle .
$$

This open state reproduces the imaginary part of the elastic amplitude at $t=0$ in the high-energy limit in eq. (2.33)

$$
\begin{aligned}
\operatorname{Im} \mathcal{A}_{g g} & =\pi \alpha^{\prime}\left\langle g_{\zeta}, \bar{g}_{\bar{\zeta}}\left|\mathcal{V}_{\overrightarrow{0}}^{\dagger} \mathcal{V}_{\overrightarrow{0}}\right| g, \bar{g}\right\rangle=\pi \alpha^{\prime} \beta^{2} \epsilon_{s}(D \bar{\epsilon})_{r} \zeta_{s^{\prime}}(D \bar{\zeta})_{r^{\prime}}\left\langle 0\left|a_{n-1}^{r^{\prime}} a_{1}^{s^{\prime}} a_{-n+1}^{r} a_{-1}^{s}\right| 0\right\rangle \\
& =\pi \alpha^{\prime} \beta^{2}(\epsilon \zeta)(\bar{\epsilon} \bar{\zeta})(n-1) \sim \pi \alpha^{\prime} \beta^{2} n(\epsilon \zeta)(\bar{\epsilon} \bar{\zeta})
\end{aligned}
$$

as one can see by using eq. (3.6).

Let us now consider the leading terms in the expansion of $\mathcal{V}_{\vec{p} t}$ for small transverse momenta and derive the absorption amplitude in impact parameter space. We shall focus on the leading term in eq. (2.34), neglecting contributions suppressed by additional powers of $\log \alpha^{\prime} s$. The steps are essentially the same as for the absorption of the tachyon. We 
first notice that as in that case the Neumann coefficients multiplying an open mode $a_{-k}$ have a dependence on the transverse momentum of the form $k^{-\frac{\alpha^{\prime}}{4} \vec{q}^{2} \frac{k}{n} \log n}$, that in impact parameter space gives again a Gaussian in $b$. We can then neglect all the terms proportional to $\vec{P}$ since they become derivatives $\partial_{b}$ and correspond to subleading terms ${ }^{14}$ in an expansion in powers of $\frac{b^{2}}{\alpha^{\prime} \log \alpha^{\prime} s}$. Therefore at high energy and for small $\lambda$ the second (dominant) term in the right-hand-side of eq. (5.36) becomes

$$
\mathcal{V}_{\vec{p}_{t}}\left|g_{\epsilon}, \bar{g}_{\bar{\epsilon}}\right\rangle \sim-\beta \mathcal{P}_{n}\left[e^{N_{k}^{3} P_{i} a_{-k}^{i}} N_{1 u}^{23}\left(\epsilon a_{-1}\right)\left(a_{-u} D \bar{\epsilon}\right)\right]|0\rangle
$$

Using that

$$
N_{1 u}^{23} \sim u^{-\lambda \frac{u}{n}}, \quad N_{k}^{3} \sim \frac{k^{-\lambda \frac{k}{n}}}{k} \sqrt{\frac{\alpha^{\prime}}{2}} \vec{p}_{t}
$$

and following the same steps as in the discussion of the tachyon, we find in impact parameter space

$$
\begin{aligned}
\widetilde{\mathcal{V}}_{\vec{b}}\left|g_{\epsilon}, \bar{g}_{\bar{\epsilon}}\right\rangle & =\frac{1}{\left(\pi \alpha^{\prime} \log \alpha^{\prime} s\right)^{\frac{24-p}{2}}} \mathcal{P}_{n}\left[e^{-\frac{1}{\alpha^{\prime} \log \alpha^{\prime} s}\left(b^{i}+i \sqrt{\frac{\alpha^{\prime}}{2}} \sum_{k} \frac{1}{k} a_{-k}^{i}\right)^{2}} \sum_{u=1}^{\infty}\left(\epsilon a_{-1}\right)\left(a_{-u} D \bar{\epsilon}\right)\right]|0\rangle \\
& =\frac{e^{-\frac{b^{2}}{2 \alpha^{\prime} \log \alpha^{\prime} s}}}{\left(\pi \alpha^{\prime} \log \alpha^{\prime} s\right)^{\frac{24-p}{4}}} \mathcal{P}_{n} \sum_{u=1}^{\infty}\left(\epsilon a_{-1}\right)\left(\bar{\epsilon} D a_{-u}\right)|b\rangle_{X}
\end{aligned}
$$

and

$$
\operatorname{Im} \mathcal{A}_{g g}=\pi \alpha^{\prime}\left\langle g_{\zeta}, \bar{g}_{\bar{\zeta}}\left|\mathcal{V}_{\overrightarrow{0}}^{\dagger} \widetilde{\mathcal{V}}_{\vec{b}}\right| g_{\epsilon}, \bar{g}_{\bar{\epsilon}}\right\rangle=(\epsilon \zeta)(\bar{\epsilon} \bar{\zeta}) \operatorname{Im} \mathcal{A}_{T T},
$$

in agreement with eq. (2.25).

\subsection{First massive level}

We turn now to the absorption of a massive string state belonging to the first massive level $N_{c}=2$, reviewed in section 2. We will discuss the absorption of a closed state of the form $(L \otimes \bar{L})$ or $(H \otimes \bar{H})$. We will see that in both cases the enhancement of the $N^{13}$ Neumann coefficients in eq. (4.16) leads to a simple form for the open state. The analysis of the absorption of $(H \otimes \bar{H})$ will also show that in the closed-open vertex at high energy while the left components of the polarization tensor always appear contracted with open modes (terms proportional to $N^{13}$ ), the right components can appear either contracted with open modes (terms proportional to $N^{23}$ ) or among themselves (terms proportional to $N^{22}$ ).

It is therefore not immediate to see how the product of two absorption amplitudes can reproduce the imaginary part of the elastic amplitude in eqs. (2.33) since the latter contains only one type of contraction, where the left (right) components of the polarization tensor of one state are contracted with the left (right) components of the other. We will argue that the coefficients of all the other types of contractions that could appear in the product indeed vanish.

\footnotetext{
${ }^{14}$ If we set $\mu=0$ before taking the limit of small transverse momentum this would not be true for the terms containing $N_{1}^{2}$ and up to two factors of $\vec{P}$, since $N_{1}^{2} \sim \frac{1}{\alpha^{\prime} \vec{p}_{t}^{2}}$. These terms would lead to a dependence of the open state on the direction along which the transverse momentum is sent to zero.
} 
The simplification of the form of the open state due to the enhancement of the $N^{13}$ Neumann coefficients and the above-mentioned cancellation are the two main new features that appear in the evaluation of the absorption of a massive closed string. The analysis of the first massive level will then make the discussion of the general case relatively straightforward.

Let us consider first the state

$$
\left|L_{\epsilon}, \bar{L}_{\bar{\epsilon}}\right\rangle=\frac{1}{2}\left(\epsilon A_{-2}\right)\left(\bar{\epsilon} \bar{A}_{-2}\right)|0\rangle .
$$

At high energy the Neumann coefficients become

$$
\begin{aligned}
N_{k l}^{33} \sim-\frac{\omega}{n} \frac{1}{k+l} \frac{k^{-\omega \frac{k}{n}}}{\Gamma\left(1-\omega \frac{k}{n}\right)} \frac{l^{-\omega \frac{l}{n}}}{\Gamma\left(1-\omega \frac{l}{n}\right)}, \quad N_{2 k}^{23} \sim \frac{n}{\omega} \frac{2}{2-\frac{\omega}{n} k} \frac{k^{-\frac{k}{n}}}{\Gamma\left(1-\frac{k}{n}\right)}, \\
N_{22}^{12} \sim-\frac{n}{\omega}, \quad N_{2 k}^{13} \sim \frac{\omega}{n(k-2)} \frac{k^{-\omega \frac{k}{n}}}{\Gamma\left(1-\omega \frac{k}{n}\right)}, \quad k \neq 2, \quad N_{2,2}^{13} \sim-\frac{1}{2}, \quad \\
N_{2}^{1} \vec{P}=\frac{1}{2} \sqrt{\frac{\alpha^{\prime}}{2}} \vec{p}_{t}, \quad N_{2}^{2} \vec{P}=-\frac{n^{2}}{\omega^{2}} \sqrt{\frac{\alpha^{\prime}}{2}} \vec{p}_{t}, \quad N_{k}^{3} \vec{P}=\frac{k^{-\omega \frac{k}{n}}}{\Gamma\left(1-\omega \frac{k}{n}\right)} \frac{1}{k} \sqrt{\frac{\alpha^{\prime}}{2}} \vec{p}_{t},
\end{aligned}
$$

where $\omega=1+\frac{\alpha^{\prime}}{4} \vec{p}_{t}^{2}$. For this example we find, limiting ourselves to $\vec{p}_{t}=0$,

$$
Z_{c, L}=N_{22}^{12} A_{2} D \bar{A}_{2}+N_{2 u}^{23} \bar{A}_{2} D a_{-u}+N_{2 u}^{13} A_{2} a_{-u},
$$

and

$$
V_{\overrightarrow{0}}\left|L_{\epsilon}, \bar{L}_{\bar{\epsilon}}\right\rangle=\beta \mathcal{P}_{n} e^{Z_{o}}\left[2(\epsilon D \bar{\epsilon}) N_{22}^{12}+2 N_{2 v}^{13} N_{2 u}^{23}\left(\epsilon a_{-v}\right)\left(a_{-u} D \bar{\epsilon}\right)\right]|0\rangle .
$$

To identify which of the terms in the square brackets in eq. (5.51) gives the leading contribution at high energy we need to keep track of the powers of the energy associated with the Neumann coefficients $N_{k l}^{33}$. The main observation is that since these coefficients scale like

$$
N_{k l}^{33} \sim n^{-\omega\left(\frac{k}{n}+\frac{l}{n}\right)-2}
$$

the insertion of $Q$ couples of open modes gives a contribution to the discontinuity of the elastic amplitude that scales like

$$
n^{-2 Q-2 \omega} n^{2 Q-1}=n^{-1-2 \omega}=n^{1-2 N_{c}-\frac{\alpha^{\prime}}{2} \vec{p}_{t}^{2}},
$$

where the power $n^{2 Q-1}$ comes from the sum over all possible states. In order for a term in the expansion of $e^{Z_{c, \psi}}$ to contribute with the leading power of the energy we then need a power of $n^{2 N_{c}}$ from the square of the corresponding Neumann coefficients and the sum over the possible states. In the case at hand we need a factor of $n^{4}$. The first term in eq. (5.51) is therefore always subleading while in the second we need to set $v=2$ so that

$$
\mathcal{V}_{\overrightarrow{0}}\left|L_{\epsilon}, \bar{L}_{\bar{\epsilon}}\right\rangle=-\beta N_{2 u}^{23} \mathcal{P}_{n}\left[e^{Z_{o}}\left(\epsilon a_{-2}\right)\left(a_{-u} D \bar{\epsilon}\right)\right]|0\rangle
$$

The form of the open state is very simple. The left part of the closed polarization tensor is contracted with open modes with the same mode number as the left closed modes, 
as a result of the enhancement of the coefficient $N_{22}^{13}$, eq. (4.16). The right part of the closed polarization tensor is contracted with open modes with mode number $u$ of order $n$ (coefficients $N_{2 u}^{23}$ ).

As a check of eq. (5.54) let us evaluate the product

$$
\left\langle L_{\zeta}, \bar{L}_{\bar{\zeta}}\left|\mathcal{V}_{\overrightarrow{0}}^{\dagger} \mathcal{V}_{\overrightarrow{0}}\right| L_{\epsilon}, \bar{L}_{\bar{\epsilon}}\right\rangle=\zeta_{s^{\prime}}(D \bar{\zeta})_{r^{\prime}} \epsilon_{s}(D \bar{\epsilon})_{r} \beta^{2} \sum_{u, v} N_{2 u}^{23} N_{2 v}^{23}\left\langle 0\left|a_{2}^{s^{\prime}} a_{v}^{r^{\prime}} e^{Z_{o}^{\dagger}} \mathcal{P}_{n} e^{Z_{o}} a_{-2}^{s} a_{-u}^{r}\right| 0\right\rangle
$$

In order to do this, we expand the exponentials and use the fact that the open modes with mode number of order $n$ in $Z_{o}$ commute with the open modes with mode number of order one. The direct contraction between the oscillators without any $N^{33}$ insertion is subleading since the sum over $u$ is then restricted to $u=n-2$ and $N_{2, n-2}^{23} \sim \frac{4}{n}$. The next term requires the matrix element

$$
\left\langle 0\left|a_{v}^{r^{\prime}} a_{k^{\prime}}^{i} a_{l^{\prime}}^{i} a_{-k}^{j} a_{-l}^{j} a_{-u}^{r}\right| 0\right\rangle,
$$

which gives two inequivalent contractions between the transverse indices. We find

$$
\operatorname{Im} \mathcal{A}_{L L} \sim \pi \alpha^{\prime} \beta^{2} n(\epsilon \zeta)(\bar{\epsilon} \bar{\zeta})\left[96 \cdot I_{L, 1}+8 \cdot I_{L, 2}\right]
$$

where the explicit expressions for the integrals $I_{L, i}, i=1,2$, can be found in appendix B together with a more detailed description of the calculation. Substituting the numerical values we obtain the first approximation to the imaginary part of the disc

$$
\operatorname{Im} \mathcal{A}_{L L} \sim \pi \alpha^{\prime} \beta^{2} n(\epsilon \zeta)(\bar{\epsilon} \bar{\zeta}) 0.841
$$

If we include the second term in the series, we obtain

$$
\operatorname{Im} \mathcal{A}_{L L} \sim \pi \alpha^{\prime} \beta^{2} n(\epsilon \zeta)(\bar{\epsilon} \bar{\zeta}) 0.994
$$

The matrix elements and the integrals required to evaluate this second term are again collected in appendix B (see eqs. (B.8), (B.9) and (B.10)).

We turn now to the state

$$
\left|H_{\epsilon}, \bar{H}_{\bar{\epsilon}}\right\rangle=\frac{1}{2} \epsilon_{(1)}^{i} \epsilon_{(2)}^{j} \bar{\epsilon}_{(1)}^{k} \bar{\epsilon}_{(2)}^{l} A_{-1}^{i} A_{-1}^{j} \bar{A}_{-1}^{k} \bar{A}_{-1}^{l}|0\rangle,
$$

with $\hat{\epsilon}^{i j} \equiv \epsilon_{(1)}^{i} \epsilon_{(2)}^{j}$ and $\hat{\bar{\epsilon}}^{k l} \equiv \bar{\epsilon}_{(1)}^{k} \bar{\epsilon}_{(2)}^{l}$ symmetric tensors. The Neumann coefficients with two open modes are the same as before while those with two closed modes are

$$
N_{11}^{11} \sim \frac{\omega}{2 n}, \quad N_{11}^{12} \sim 1, \quad N_{11}^{22} \sim \frac{n^{2}}{2 \omega^{2}} .
$$

The mixed open-closed coefficients are

$$
\begin{aligned}
& N_{1 k}^{23} \sim \frac{1}{1-\omega \frac{k}{n}} \frac{k^{-\omega \frac{k}{n}}}{\Gamma\left(1-\omega \frac{k}{n}\right)}, \quad k \neq n, \quad N_{1 n}^{23} \sim \frac{1}{n}, \quad k=n, \\
& N_{1 k}^{13} \sim-\frac{\omega}{n(k-1)} \frac{k^{-\omega \frac{k}{n}}}{\Gamma\left(1-\omega \frac{k}{n}\right)}, \quad k \neq 1, \quad N_{11}^{13} \sim 1 .
\end{aligned}
$$


Finally

$$
N_{1}^{1} \vec{P}=-\sqrt{\frac{\alpha^{\prime}}{2}} \vec{p}_{t}, \quad N_{1}^{2} \vec{P}=-\frac{n}{\omega} \sqrt{\frac{\alpha^{\prime}}{2}} \vec{p}_{t}, \quad N_{k}^{3} \vec{P}=\frac{k^{-\omega \frac{k}{n}}}{\Gamma\left(1-\omega \frac{k}{n}\right)} \frac{1}{k} \sqrt{\frac{\alpha^{\prime}}{2}} \vec{p}_{t} .
$$

Let us discuss explicitly only the vertex with $\vec{p}_{t}=0$. We need to retain the following closed modes:

$$
Z_{c, H}=\frac{1}{2} N_{11}^{11} A_{1}^{r} A_{1}^{r}+\frac{1}{2} N_{11}^{22} \bar{A}_{1}^{r} \bar{A}_{1}^{r}+N_{11}^{12} A_{1}^{r} D \bar{A}_{1}^{r}+N_{1 u}^{23} \bar{A}_{1}^{r} D a_{-u}^{r}+N_{1 u}^{13} A_{1}^{r} a_{-v}^{r},
$$

and terms up to the forth order in the expansion of $e^{Z_{c, H}}$ can contribute to the absorption amplitude. We find

$$
\begin{aligned}
V_{\overrightarrow{0}}\left|H_{\epsilon}, \bar{H}_{\bar{\epsilon}}\right\rangle= & \beta \mathcal{P}_{n} e^{Z_{o}}\left[\left(N_{11}^{12}\right)^{2}\left(\epsilon_{(1)} D \bar{\epsilon}_{(1)}\right)\left(\epsilon_{(2)} D \bar{\epsilon}_{(2)}\right)+\frac{1}{2} N_{11}^{11} N_{11}^{22}\left(\epsilon_{(1)} \epsilon_{(2)}\right)\left(\bar{\epsilon}_{(1)} \bar{\epsilon}_{(2)}\right)\right. \\
& +\frac{1}{2} N_{11}^{22} N_{1 u}^{13} N_{1 v}^{13} \epsilon_{(1)}^{s} \epsilon_{(2)}^{t}\left(\bar{\epsilon}_{(1)} \bar{\epsilon}_{(2)}\right) a_{-u}^{s} a_{-v}^{t} \\
& +\frac{1}{2} N_{11}^{11} N_{1 u}^{23} N_{1 v}^{23}\left(\epsilon_{(1)} \epsilon_{(2)}\right)\left(D \bar{\epsilon}_{(1)}\right)^{s}\left(D \bar{\epsilon}_{(2)}\right)^{t} a_{-u}^{s} a_{-v}^{t} \\
& +2 N_{11}^{12} N_{1 u}^{23} N_{1 v}^{13}\left(\epsilon_{(1)} D \bar{\epsilon}_{(1)}\right) \epsilon_{(2)}^{t}(D \bar{\epsilon})_{(2)}^{s} a_{-u}^{s} a_{-v}^{t} \\
& \left.+\frac{1}{2} N_{1 u}^{23} N_{1 v}^{23} N_{1 w}^{13} N_{1 z}^{13} \epsilon_{(1)}^{t} \epsilon_{(2)}^{y}(D \bar{\epsilon})_{(1)}^{r}(D \bar{\epsilon})_{(2)}^{s} a_{-u}^{r} a_{-v}^{s} a_{-w}^{t} a_{-z}^{y}\right]|0\rangle
\end{aligned}
$$

Let us analyze this expression and show that the leading contribution at high energy is due to the first term in the second line and to the last term. Substituting the explicit values of the Neumann coefficients in the first two terms we find contributions of the form

$$
e^{Z_{o}}\left[\left(\epsilon_{(1)} D \bar{\epsilon}_{(1)}\right)\left(\epsilon_{(2)} D \bar{\epsilon}_{(2)}\right)+\frac{n}{4}\left(\epsilon_{(1)} \epsilon_{(2)}\right)\left(\bar{\epsilon}_{(1)} \bar{\epsilon}_{(2)}\right)\right]|0\rangle,
$$

which are clearly subleading. The next three terms are

$$
\begin{aligned}
e^{Z_{o}} & {\left[\frac{n^{2}}{4} N_{1 u}^{13} N_{1 v}^{13}\left(\bar{\epsilon}_{(1)} \bar{\epsilon}_{(2)}\right) \epsilon_{(1)}^{s} \epsilon_{(2)}^{t} a_{-u}^{s} a_{-v}^{t}+\frac{1}{4 n} N_{1 u}^{23} N_{1 v}^{23}\left(\epsilon_{(1)} \epsilon_{(2)}\right)\left(D \bar{\epsilon}_{(1)}\right)^{s}\left(D \bar{\epsilon}_{(2)}\right)^{t} a_{-u}^{s} a_{-v}^{t}\right.} \\
& \left.+2 N_{1 u}^{23} N_{1 v}^{13}\left(\epsilon_{(1)} D \bar{\epsilon}_{(1)}\right) \epsilon_{(2)}^{t}\left(D \bar{\epsilon}_{(2)}\right)^{s} a_{-u}^{s} a_{-v}^{t}\right]|0\rangle .
\end{aligned}
$$

For generic values of the indices they are subleading but when both $N^{13}$ factors are enhanced the first term scales with the power of the energy required to be relevant in the high energy limit. Finally also the term with four open modes gives a leading contribution only when both the $N^{13}$ factors are enhanced. The result of this analysis is that

$$
\begin{aligned}
\mathcal{V}_{\overrightarrow{0}}\left|H_{\epsilon}, \bar{H}_{\bar{\epsilon}}\right\rangle= & \beta \mathcal{P}_{n} e^{Z_{o}}\left[\frac{1}{2} \sum_{u, v} N_{1 u}^{23} N_{1 v}^{23} \epsilon_{(1)}^{s} \epsilon_{(2)}^{t}\left(D \bar{\epsilon}_{(1)}\right)^{r}\left(D \bar{\epsilon}_{(2)}\right)^{l} a_{-1}^{s} a_{-1}^{t} a_{-u}^{r} a_{-v}^{l}\right. \\
& \left.+\frac{n^{2}}{4}\left(\bar{\epsilon}_{(1)} \bar{\epsilon}_{(2)}\right) \epsilon_{(1)}^{s} \epsilon_{(2)}^{t} a_{-1}^{s} a_{-1}^{t}\right]|0\rangle .
\end{aligned}
$$

The open state has again a simple form. As before, the left part of the closed polarization tensor is contracted with open modes that coincide with the left closed modes, as a result 
of the enhancement of the coefficient $N_{11}^{13}$, eq. (4.16). However the indices of the right part of the closed polarization tensor can be contracted either with open modes of order $n$ (coefficients $N_{1 u}^{23}$ ) or among themselves (coefficient $N_{11}^{22}$ ). The latter terms give additional contributions to the closed-open couplings of closed states with non-vanishing traces for their right polarization tensor.

Let us now consider the imaginary part of the elastic amplitude. We proceed as we did for the tachyon, expanding the exponentials $e^{Z_{o}}$ and evaluating the first few terms in the series to show that they already account for most of the discontinuity. We shall use again the fact that low frequency open modes commute with the high frequency modes in $Z_{o}$. We find

$$
\begin{aligned}
\operatorname{Im} \mathcal{A}_{H H} \sim & \pi \alpha^{\prime} \beta^{2}\left(\hat{\epsilon}_{i j} \hat{\zeta}_{i j}\right) \frac{1}{8}\langle 0|\left[n^{2} \hat{\bar{\zeta}}_{l l}+2 N_{1 u^{\prime}}^{23} N_{1 v^{\prime}}^{23}(D \hat{\bar{\zeta}} D)_{r^{\prime} w^{\prime}} a_{u^{\prime}}^{r^{\prime}} a_{v^{\prime}}^{w^{\prime}}\right] e^{Z_{o}^{\dagger}} \\
& \mathcal{P}_{n} e^{Z_{o}}\left[n^{2} \hat{\bar{\epsilon}}_{k k}+2 N_{1 u}^{23} N_{1 v}^{23}(D \hat{\bar{\epsilon}} D)_{r w} a_{-u}^{r} a_{-v}^{w}\right]|0\rangle
\end{aligned}
$$

The first non-vanishing term in the series is

$$
\left(\hat{\epsilon}_{i j} \hat{\zeta}_{i j}\right)\left(\hat{\bar{\epsilon}}_{k l} \hat{\bar{\zeta}}_{k l}\right) \sum_{u, v} \delta_{u+v, n}\left(N_{1 u}^{23} N_{1 v}^{23}\right)^{2} u v \sim\left(\hat{\epsilon}_{i j} \hat{\zeta}_{i j}\right)\left(\hat{\bar{\epsilon}}_{k l} \hat{\bar{\zeta}}_{k l}\right) n \int_{0}^{1} d x \frac{x^{1-2 x}(1-x)^{-1+2 x}}{\Gamma^{2}(2-x) \Gamma^{2}(1+x)} .
$$

The numerical value of the integral is

$$
I_{H, 1}=\int_{0}^{1} d x \frac{x^{1-2 x}(1-x)^{-1+2 x}}{\Gamma^{2}(2-x) \Gamma^{2}(1+x)} \sim 0.831797
$$

and we see that, as in the previous cases, the first term already accounts for most of the discontinuity. However, as shown in eq. (B.13) and also discussed below, the other two contributions in eq. (5.69) turn out to give contractions among the polarizations that are not present in the imaginary part of the amplitude. In order to see if they cancel, we have included higher order terms that come from the expansion of the exponentials in eq. (5.69). The detailed derivation of them is given in appendix B. The result is

$$
\begin{aligned}
\operatorname{Im} \mathcal{A}_{H H} \sim & \pi \alpha^{\prime} \beta^{2} n\left(\hat{\epsilon}_{i j} \hat{\zeta}_{i j}\right)\left[\left(I_{H, 1}+12 I_{H, 4}+2 I_{H, 5}\right)\left(\hat{\bar{\epsilon}}_{k l} \hat{\bar{\zeta}}_{k l}\right)\right. \\
& \left.+\left(-\frac{1}{2} I_{H, 2}+\frac{3}{2} I_{H, 3}+\frac{1}{2} I_{H, 6}-6 I_{H, 7}-\frac{1}{2} I_{H, 8}+9 I_{H, 9}+\frac{3}{4} I_{H, 10}\right)\left(\hat{\bar{\epsilon}}_{k k} \hat{\bar{\zeta}}_{l l}\right)\right],
\end{aligned}
$$

where the explicit expressions for the integrals $I_{H, i}$ and their numerical values are collected in the appendix. The main new feature is that the imaginary part seems to be proportional to two different contractions of the polarization tensors

$$
\left(\hat{\epsilon}_{i j} \hat{\zeta}_{i j}\right)\left(\hat{\bar{\epsilon}}_{k l} \hat{\bar{\zeta}}_{k l}\right), \quad\left(\hat{\epsilon}_{i j} \hat{\zeta}_{i j}\right) \hat{\bar{\epsilon}}_{k k} \hat{\bar{\zeta}}_{l l}
$$

The first contraction is the only one we expect according to the discussion in section 2 and therefore the coefficient of the second one should vanish. A cancellation is indeed possible since the coefficient of the contraction $\hat{\bar{\epsilon}}_{k k} \hat{\bar{\zeta}}_{l l}$ receives contributions from terms with opposite sign according to whether they contain an even or an odd number of coefficients $N_{k l}^{33}$. We 
do not have a general proof that the cancellation actually occurs but the explicit evaluation of the higher order terms in eq. (5.72) seems to indicate that this is the case since the sum of all the $I_{H, i}$ in the second line of eq. (5.72) gives a result which is one order of magnitude smaller than the individual terms. Indeed, substituting the numerical values of the integrals in eq. (5.72) we find

$$
\operatorname{Im} \mathcal{A}_{H H} \sim \pi \alpha^{\prime} \beta^{2} n\left(\hat{\epsilon}_{i j} \hat{\zeta}_{i j}\right)\left[0.993\left(\hat{\bar{\epsilon}}_{k l} \hat{\bar{\zeta}}_{k l}\right)-0.004\left(\hat{\bar{\epsilon}}_{k k} \hat{\bar{\zeta}}_{l l}\right)\right] .
$$

It would be interesting to have a general proof of this cancellation to all orders in the series expansion.

So far our discussion of the closed-open vertex for the states of the first massive level has been limited to $\vec{p}_{t}=0$. As we did for the tachyon and the massless sector, we can derive the vertex in impact parameter space from the small $\vec{p}_{t}$ behaviour of the vertex in momentum space. The steps are the same and the result is again that the vertex in impact parameter space is given by the vertex at zero momentum multiplied by the squeezed state in the effective modes (5.23). It is easy to check that it reproduces the leading term in the imaginary part of the elastic amplitude, eq. (2.34).

\subsection{Generic massive states}

The pattern observed in our study of the first massive level generalizes to arbitrary mass levels, with the only requirement that the closed state be ultrarelativistic, $M \ll E$. We will find that, thanks to the properties of the Neumann coefficients discussed in section 4 , even in this more general case it is still possible to give a simple, explicit and systematic description of the open state created in the $s$-channel. Consider a closed state $|\psi\rangle$ at level $N_{c}$

$$
|\psi\rangle=\prod_{k=1}^{\infty} \frac{1}{\sqrt{n_{k} ! k^{n_{k}}}}\left(\epsilon_{(k)} A_{-k}\right)^{n_{k}} \prod_{l=1}^{\infty} \frac{1}{\sqrt{\bar{n}_{l} ! l^{\bar{n}_{l}}}}\left(\bar{\epsilon}_{(l)} \bar{A}_{-l}\right)^{\bar{n}_{l}}|0\rangle,
$$

created by a collection of left and right modes, $A_{-k}^{i_{k}}$ and $\bar{A}_{-l}^{j_{l}}$, with multiplicity $n_{k}$ and $\bar{n}_{l}$ such that

$$
\sum_{\bar{k}=1}^{\infty} k n_{k}=\sum_{\bar{l}=1}^{\infty} l \bar{n}_{l}=N_{c}
$$

and characterized by a collection of left and right polarization vectors $\epsilon_{\left(k_{\alpha}\right)}^{i_{\alpha}}, \bar{\epsilon}_{\left(l_{\beta}\right)}^{j_{\beta}}$ with $\alpha=1, \ldots, n_{k}$ and $\beta=1, \ldots, \bar{n}_{l}$. Here we are using the compact notation

$$
\left(\epsilon_{(k)} A_{-k}\right)^{n_{k}} \equiv \prod_{\alpha=1}^{n_{k}} \epsilon_{\left(k_{\alpha}\right)}^{i_{\alpha}} A_{-k}^{i_{\alpha}}
$$

The action of the closed-open vertex on this state gives

$$
\mathcal{V}_{\overrightarrow{0}}|\psi\rangle=\beta \mathcal{P}_{n} e^{Z_{o}+Z_{c, \psi}}|\psi\rangle
$$

where, as before, $Z_{o}$ contains only open string modes while $Z_{c, \psi}$ both open and closed string modes, the latter restricted to those in the set used to define the closed state. 
Consider first the vertex with $\vec{p}_{t}=0$. The action of $e^{Z_{c, \psi}}$ on the state gives a polynomial in the open modes of the same order as the monomial in the closed modes that defines the closed state. From the high-energy scaling of the Neumann coefficients discussed in section 4 , it follows that the leading contributions to the absorption amplitude are obtained when the indices of the open and closed modes in the coefficients $N_{k u}^{13}$ coincide. The right closed modes are then either coupled to open modes of order $n$ through the coefficients $N_{l u}^{23}$ or contracted among themselves using the coefficients $N_{k l}^{22}$. Finally one acts on the resulting polynomial with the operator $e^{Z_{o}}$. The first few terms in the expansion of the exponential already give an accurate description of the open state.

We can then write the following general formula for the open state created on the brane world-volume when the closed state $|\psi\rangle$ is absorbed by the $\mathrm{D} p$-brane system:

$$
\begin{aligned}
\mathcal{V}_{\overrightarrow{0}}|\psi\rangle= & \beta \mathcal{P}_{n} e^{Z_{o}} \prod_{k=1}^{\infty} \sqrt{\frac{k^{n_{k}}}{n_{k} !}}\left(N_{k k}^{13}\right)^{n_{k}}\left(\epsilon_{(k)} a_{-k}\right)^{n_{k}}\left[\prod_{l=1}^{\infty} \sqrt{\frac{l^{\bar{n}_{l}}}{\bar{n}_{l} !}} \prod_{\beta=1}^{\bar{n}_{l}} N_{l u_{\beta}}^{23} D \bar{\epsilon}_{\left(l_{\beta}\right)}^{j_{\beta}} a_{-u_{\beta}}^{j_{\beta}}\right. \\
& +\sum_{r=1}^{\infty} \frac{\bar{n}_{r}}{2}\left(\bar{n}_{r}-1\right) N_{r r}^{22}\left(\bar{\epsilon}_{\left(r_{1}\right)} \bar{\epsilon}_{\left(r_{2}\right)}\right) \sqrt{\frac{r^{\bar{n}_{r}}}{\bar{n}_{r} !}} \prod_{\rho=3}^{\bar{n}_{r}} N_{r u_{\rho}}^{23} D \bar{\epsilon}_{\left(r_{\rho}\right)}^{j_{\rho}} a_{-u_{\rho}}^{j_{\rho}} \prod_{\substack{l=1 \\
l \neq r}}^{\infty} \sqrt{\frac{l_{\bar{n}_{l}}}{\bar{n}_{l} !}} \prod_{\beta=1}^{\bar{n}_{l}} N_{l u_{\beta}}^{23} D \bar{\epsilon}_{\left(l_{\beta}\right)}^{j_{\beta}} a_{-u_{\beta}}^{j_{\beta}} \\
& +\sum_{\substack{r, s=1 \\
r \neq s}}^{\infty} \bar{n}_{r} \bar{n}_{s} N_{r s}^{22}\left(\bar{\epsilon}_{\left(r_{1}\right)} \bar{\epsilon}_{\left(s_{1}\right)}\right) \sqrt{\frac{r^{\bar{n}_{r}}}{\bar{n}_{r} !}} \prod_{\rho=2}^{\bar{n}_{r}} N_{r u_{\rho}}^{23} D \bar{\epsilon}_{\left(r_{\rho}\right)}^{j_{\rho}} a_{-u_{\rho}}^{j_{\rho}} \sqrt{\frac{s^{\bar{n}_{s}}}{\bar{n}_{s} !} \prod_{\sigma=2}^{\bar{n}_{s}} N_{s u_{\sigma}}^{23} D \bar{\epsilon}_{\left(r_{\sigma}\right)}^{j_{\sigma}} a_{-u_{\sigma}}^{j_{\sigma}}} \\
& \left.\prod_{\substack{l=1 \\
l \neq r, s}}^{\infty} \sqrt{\frac{l_{\bar{n}}}{\bar{n}_{l} !}} \prod_{\beta=1}^{\bar{n}_{l}} N_{l u_{\beta}}^{23} D \bar{\epsilon}_{\left(l_{\beta}\right)}^{j_{\beta}} a_{-u_{\beta}}^{j_{\beta}}+\ldots .79\right)
\end{aligned}
$$

where the dots stand for all the other possible pairings of the right closed modes. Although notationally cumbersome, the previous formula neatly summarizes the representation of the massive open state in our basis of light-cone modes. There is a subset of open modes that are contracted with the left polarization vectors and copy precisely the left part of the closed state, in that they carry exactly the same indices. There are then open modes of order $n$ contracted with the right polarization vectors, plus all possible terms that can be obtained by contracting couples of right polarization indices among themselves. Finally there are insertions of traces of open modes $a_{-u}^{i} a_{-v}^{i}$ from the expansion of the operator $e^{Z_{o}}$, as for the absorption of a closed tachyon.

We emphasize again that when one evaluates the imaginary part of the disk using the closed-open couplings given by eq. (5.79) only the term proportional to the contraction

$$
\prod_{(k, \alpha)}\left(\zeta_{\left(k_{\alpha}\right)} \epsilon_{\left(k_{\alpha}\right)}\right) \prod_{(l, \beta)}\left(\bar{\zeta}_{l_{\beta}} \bar{\epsilon}_{l_{\beta}}\right)
$$

between the polarizations of the initial and the final state should remain, while all the terms containing one or more factors of the form

$$
\left(\bar{\zeta}_{\left(r_{\rho}\right)} \bar{\zeta}_{\left(s_{\sigma}\right)}\right)\left(\bar{\epsilon}_{\left(r_{\rho^{\prime}}\right)} \bar{\epsilon}_{\left(s_{\sigma^{\prime}}\right)}\right)
$$

should cancel. We do not have a general proof that this is the case. 
Finally, the vertex in impact parameter space is given by the vertex at zero momentum multiplied by the squeezed state in the effective modes in eq. (5.23) and reproduces the leading term in the imaginary part of the elastic amplitude, eq. (2.34).

\section{Conclusions}

In this paper we have started the analysis of the absorption of a light, very energetic string by a stack of $\mathrm{D} p$-branes and of the consequent excitation of the latter. The final aim of this study is to show how such a complicated process can be described in terms of a unitary $S$-matrix, thereby generalizing to the absorption regime what has been achieved so far in the scattering regime (including tidal excitation of the closed string).

This problem, in general, is a very complicated one. It becomes tractable, however, by appropriately restricting the kinematic regime under scrutiny as explained in detail in the introduction. This allows, on the one hand, to ignore closed-string loops (and therefore gravitational bremsstrahlung) and, on the other hand, to neglect higher corrections to the leading eikonal. Under these approximations one expects to be able to construct a unitary $S$-matrix in a Hilbert space consisting of a single (but possibly excited) closed string and of an arbitrary number of open strings living on the brane world-volume.

Here we have considered the first term of this eikonal resummation, namely the treelevel (disk) approximation, postponing to further work [20] the full unitarization program. In this approximation also the open-string Hilbert space contains a single (in general highly excited) string. A very encouraging outcome of our analysis has been the emergence of a simple description of the relevant states in the open sector. This makes one hope that resummation of the eikonal series will not be that hard. At the same time, the simple properties of the open sector can be given a classical (or semi-classical) interpretation [14], suggesting that a semiclassical treatment of the whole series could be sufficient for yielding a unitary $S$-matrix.

We conclude by mentioning that the price to pay for making the problem tractable is to work at sufficiently small string coupling for the characteristic radius of the brane-induced geometry $R_{p}$ to be smaller than the string length $l_{s} \sim \sqrt{\alpha^{\prime}}$ enhanced by a logarithmic factor. In that limit we expect the dominantly produced open strings to be heavy. Making contact with the AdS/CFT correspondence [1-3] in the supergravity approximation will unfortunately require the opposite limit of a large $R_{p}$ in string units. Such a regime appears still far from what our present computational technology can achieve.

\section{Acknowledgements}

G.D. thanks the Galileo Galilei Institute for Theoretical Physics for hospitality and the INFN for partial support while this research was being carried out. G.D. also gratefully acknowledges the hospitality of the Department of Applied Mathematics and Theoretical Physics (Cambridge) at various times during the completion of this work. P. D. thanks C. Maccaferri for discussions on the open-closed string vertex. The research of $\mathrm{R}$. $\mathrm{R}$. is partially supported by STFC (Grant ST/L000415/1, String theory, gauge theory 8 duality). G.V. 
would like to acknowledge the hospitality of the Kavli Institute for Theoretical Physics, University of California, Santa Barbara (research supported in part by the National Science Foundation under Grant No. NSF PHY11-25915) where part of this research has been carried out.

\section{A The light-cone vertex and the covariant amplitudes}

In this appendix we make a direct comparison between the closed-open transition amplitudes evaluated using the light-cone vertex and those derived using the covariant formalism. The simplest closed-open transition amplitudes that can be evaluated using the covariant formalism are those to open states belonging to the leading Regge trajectory whose vertex operators are

$$
\mathcal{V}_{S_{n}}=\frac{g_{o}}{\sqrt{n !}}\left[\frac{-i}{\sqrt{2 \alpha^{\prime}}}\right]^{n} \prod_{i=1}^{n} \zeta_{\alpha_{i}} \partial X^{\alpha_{i}} e^{i p_{o} X}, \quad \alpha^{\prime} m^{2}=n-1,
$$

where we follow the notation introduced in sections 2 and 3. Since transitions to the tachyon and the vector are forbidden by the kinematics we only need $n \geq 2$.

As in section 3, when we evaluate an amplitude using the covariant formalism we write

$$
p_{c}=\left(E, \overrightarrow{0}_{p}, \vec{p}_{c}\right)
$$

where $\vec{p}_{c}$ belongs to the $(25-p)$-dimensional space transverse to the branes. We also define the tensor $\delta_{\perp}$ as the Kronecker delta in the spatial directions orthogonal to $\hat{p}_{c}$, where $\hat{p}_{c}$ is the unit vector $\hat{p}_{c}=\frac{\vec{p}_{c}}{\left|\vec{p}_{c}\right|}$.

When we evaluate an amplitude using the closed-open vertex we write

$$
p_{c}=\left(E, \overrightarrow{0}_{p}, \vec{p}_{t}, p\right) \text {. }
$$

Here $\vec{p}_{t}$ belongs to the $(24-p)$-dimensional space transverse to the branes and to the direction $x^{25} \equiv z$.

\section{A.1 Tachyon to leading Regge}

When the initial closed state is a tachyon we find

$$
B_{T, S_{n}}\left(\hat{p}_{c}\right)=\beta \frac{(-1)^{n}}{\sqrt{n !}}\left(\frac{n+3}{2}\right)^{\frac{n}{2}} \prod_{i=1}^{n}\left(\zeta_{i} \hat{p}_{c}\right)
$$

where we used that $\alpha^{\prime} \vec{p}_{c}{ }^{2}=n+3$. Only the polarization with all indices longitudinal is excited. For instance for $n=2$ the polarization tensor of the state that is excited is

$$
\zeta_{\text {lon }, 2}=\frac{1}{5 \sqrt{24}}\left(-\delta_{\perp}+24 \hat{p}_{c} \hat{p}_{c}\right)
$$

and for $n=3$

$$
\zeta_{\text {lon }, 3}=\frac{1}{9 \sqrt{8}}\left(\delta_{\perp} \hat{p}_{c}+\delta_{\perp} \hat{p}_{c}+\delta_{\perp} \hat{p}_{c}-24 \hat{p}_{c} \hat{p}_{c} \hat{p}_{c}\right)
$$


At level $n=2$ and $n=3$ the states of the leading Regge trajectory give all the physical states and therefore we can use the amplitudes in eq. (A.4) to reconstruct the discontinuity of the elastic amplitude at the corresponding energies. Consider the case $n=2$. To evaluate the imaginary part of the elastic amplitude we write

$$
\begin{aligned}
\operatorname{Im} A & =\pi \alpha^{\prime} \sum_{\zeta} B_{T, S_{2}}\left(\hat{p}_{1}\right) B_{T, S_{2}}\left(\hat{p}_{2}\right)=\pi \alpha^{\prime} \beta^{2} \frac{25}{8} \sum_{\zeta}\left(\zeta_{i j} \hat{p}_{1}^{i} \hat{p}_{1}^{j}\right)\left(\zeta_{k l} \hat{p}_{2}^{k} \hat{p}_{2}^{l}\right) \\
& =\pi \alpha^{\prime} \beta^{2} \frac{25}{8}\left[\left(\hat{p}_{1} \hat{p}_{2}\right)^{2}-\frac{1}{25}\right]=\frac{\pi \alpha^{\prime} \beta^{2}}{8}\left[25 \cos ^{2} \theta-1\right]=\pi \alpha^{\prime} \beta^{2}\left(3+\frac{5}{2} y+\frac{y^{2}}{2}\right)
\end{aligned}
$$

where $\theta$ is the angle between $\hat{p}_{1}$ and $\hat{p}_{2}$. The result agrees with eq. (2.4). We used that the sum over a complete set of traceless symmetric tensors of rank two in 25 spatial dimensions gives

$$
\sum_{\zeta} \zeta_{i j} \zeta_{k l}=\frac{1}{2}\left(\delta_{i k} \delta_{j l}+\delta_{i l} \delta_{j k}\right)-\frac{1}{25} \delta_{i j} \delta_{k l}
$$

and that

$$
\sin ^{2} \frac{\theta}{2}=-\frac{y}{\alpha^{\prime}\left(E^{2}-M^{2}\right)}, \quad y=\frac{\alpha^{\prime}}{4} t .
$$

The cases $n \geq 3$ can be treated in a similar way.

Let us now study the transition from the tachyon to the first massive level using the light-cone vertex. The closed-open vertex gives in this case

$$
V_{\vec{p}_{t}}|0\rangle=\beta\left[\frac{1}{2} N_{11}^{33} a_{-1}^{i} a_{-1}^{i}+\sqrt{\frac{\alpha^{\prime}}{2}} N_{2}^{3} \alpha_{3} p_{t}^{i} a_{-2}^{i}+\frac{\alpha^{\prime}}{4}\left(N_{1}^{3} \alpha_{3}\right)^{2} p_{t}^{i} p_{t}^{j} a_{-1}^{i} a_{-1}^{j}\right]|0\rangle,
$$

where

$$
N_{11}^{33}=-\frac{1-\rho^{2}}{8}, \quad N_{2}^{3} \alpha_{3}=\frac{\rho}{2}, \quad N_{1}^{3} \alpha_{3}=1, \quad \rho \equiv \frac{p}{E}=\sqrt{5-\alpha^{\prime} \vec{p}_{t}^{2}} .
$$

More explicitly

$$
V_{\vec{p}_{t}}|0\rangle=\beta\left[\frac{4-\alpha^{\prime} \vec{p}_{t}^{2}}{16} a_{-1}^{i} a_{-1}^{i}+\frac{\sqrt{5-\alpha^{\prime} \vec{p}_{t}^{2}}}{2} \sqrt{\frac{\alpha^{\prime}}{2}} p_{t}^{i} a_{-2}^{i}+\frac{\alpha^{\prime}}{4} p_{t}^{i} p_{t}^{j} a_{-1}^{i} a_{-1}^{j}\right]|0\rangle .
$$

Therefore the covariant state with the polarization tensor in eq. (A.5) corresponds to the normalized state

$$
\left|\zeta_{\text {lon }, 2}, \vec{p}_{t}\right\rangle=\frac{1}{\sqrt{3}}\left[\frac{4-\alpha^{\prime} \vec{p}_{t}^{2}}{16} a_{-1}^{i} a_{-1}^{i}+\frac{\sqrt{5-\alpha^{\prime} \vec{p}_{t}^{2}}}{2} \sqrt{\frac{\alpha^{\prime}}{2}} p_{t}^{i} a_{-2}^{i}+\frac{\alpha^{\prime}}{4} p_{t}^{i} p_{t}^{j} a_{-1}^{i} a_{-1}^{j}\right]|0\rangle,
$$

which for $\vec{p}_{t}=0$ (i.e. when the light-cone gauge is aligned to $\hat{p}_{c}$ ) reduces to

$$
\left|\zeta_{\mathrm{lon}, 2}, 0\right\rangle=\frac{1}{\sqrt{48}} a_{-1}^{i} a_{-1}^{i}|0\rangle
$$


It is amusing to verify that the state in eq. (A.13) can be obtained from the state in eq. (A.14) by a rotation of an angle $\varphi$ with $\sin \varphi=\frac{\left|\vec{p}_{t}\right|}{\left|\vec{p}_{c}\right|}$ in the plane $\left(\hat{p}_{c}, \hat{p}_{t}\right)$, as it should. If we call the rotation plane the plane $(z, y)$, the rotation operator is

$$
R_{z y}(\varphi)=e^{-i \varphi J^{z y}}, \quad J^{z y}=\frac{i}{2 \sqrt{\alpha^{\prime}}\left|p_{o}^{+}\right|} \sum_{k=1}^{\infty} \frac{1}{k}\left(a_{-k}^{y} L_{k}-L_{-k} a_{k}^{y}\right),
$$

where $L_{k}=\frac{1}{2} \sum_{l \neq 0} a_{k-l}^{i} a_{l}^{i}$. Let us consider for simplicity an infinitesimal rotation. When $\varphi \ll 1$ we find from eq. (A.13)

$$
\left|\zeta_{\text {lon }, 2}, \vec{p}_{t}\right\rangle \sim \frac{1}{4 \sqrt{3}}\left[a_{-1}^{i} a_{-1}^{i}+5 \sqrt{2} \varphi a_{-2}^{y}\right]|0\rangle .
$$

The term linear in $\varphi$ coincides with the effect of an infinitesimal rotation on the state with $\vec{p}_{t}=0$

$$
\begin{aligned}
& -i \varphi J^{z y}\left|\zeta_{\text {lon }, 2}, 0\right\rangle=\frac{1}{4 \sqrt{3}} \frac{\varphi}{\sqrt{2}} \sum_{k=1}^{\infty} \frac{1}{k}\left[a_{-k}^{y} L_{k}-L_{-k} a_{k}^{y}, a_{-1}^{i} a_{-1}^{i}\right]|0\rangle \\
= & \frac{1}{4 \sqrt{3}} \frac{\varphi}{\sqrt{2}}\left(\frac{1}{2}\left[a_{-2}^{y} L_{2}, a_{-1}^{i} a_{-1}^{i}\right]-2 L_{-1} a_{-1}^{y}\right)|0\rangle=\frac{1}{4 \sqrt{3}} \frac{\varphi}{\sqrt{2}}(12-2) a_{-2}^{y}|0\rangle .
\end{aligned}
$$

We can finally reconstruct the imaginary part of the elastic amplitude for $\alpha^{\prime} E^{2}=1$. Since we are not in the high energy limit we work in the brick wall frame defined in eq. (3.28). Using eq. (3.30) we find

$$
\begin{aligned}
\operatorname{Im} A & =\pi \alpha^{\prime} \beta^{2}\left[12\left(N_{11}^{33}\right)^{2}+\frac{y^{2}}{8}\left(N_{1}^{3} \alpha_{3}\right)^{4}-\frac{y}{2} N_{11}^{33}\left(N_{1}^{3} \alpha_{3}\right)^{2}-y\left(N_{2}^{3} \alpha_{3}\right)^{2}\right] \\
& =\pi \alpha^{\prime} \beta^{2}\left(3+\frac{5}{2} y+\frac{y^{2}}{2}\right)
\end{aligned}
$$

in agreement with eq. (2.4).

\section{A.2 Massless to leading Regge}

When the initial closed state is a massless state with vertex operator

$$
\mathcal{V}_{g}=-\frac{\kappa}{2 \pi} \frac{2}{\alpha^{\prime}} \epsilon_{\mu} \bar{\epsilon}_{\nu} \partial X^{\mu} \bar{\partial} X^{\nu} e^{i p_{c} X}, \quad \alpha^{\prime} M^{2}=0
$$

we find

$$
\begin{aligned}
B_{g, S_{n}}= & -\beta \frac{1}{\sqrt{n !}} \frac{2}{\alpha^{\prime}}\left[\frac{-i}{\sqrt{2 \alpha^{\prime}}}\right]^{n}|z-\bar{z}|^{2-n}|z-x|^{2 n} \epsilon_{\mu} \bar{\epsilon}_{\nu}\left\{-\frac{\alpha^{\prime}}{2} D^{\mu \nu} \frac{1}{(z-\bar{z})^{2}} L_{n}\right. \\
& -i \alpha^{\prime} \frac{D^{\nu \alpha_{1}} \zeta_{\alpha_{1}}}{(\bar{z}-x)^{2}}\left(\frac{\alpha^{\prime}}{2} \frac{D p_{c}^{\mu}}{z-\bar{z}}+\alpha^{\prime} \frac{p_{o}^{\mu}}{z-x}\right) n L_{n-1}+i \alpha^{\prime} \frac{\eta^{\mu \alpha_{1}} \zeta_{\alpha_{1}}}{(z-x)^{2}}\left(\frac{\alpha^{\prime}}{2} \frac{D p_{c}^{\nu}}{z-\bar{z}}+\alpha^{\prime} \frac{p_{o}^{\nu}}{x-\bar{z}}\right) n L_{n-1} \\
& \left.+\alpha^{\prime 2} \frac{D^{\nu \alpha_{1}} \zeta_{\alpha_{1}} \eta^{\mu \alpha_{2}} \zeta_{\alpha_{2}}}{(z-x)^{2}(\bar{z}-x)^{2}} n(n-1) L_{n-2}+\left(\frac{\alpha^{\prime}}{2} \frac{D p_{c}^{\mu}}{z-\bar{z}}+\alpha^{\prime} \frac{p_{o}^{\mu}}{z-x}\right)\left(\frac{\alpha^{\prime}}{2} \frac{D p_{c}^{\nu}}{z-\bar{z}}+\alpha^{\prime} \frac{p_{o}^{\nu}}{x-\bar{z}}\right) L_{n}\right\},
\end{aligned}
$$

where

$$
L_{k}=\left(\frac{z-\bar{z}}{|x-z|^{2}}\right)^{k} \prod_{i=n-k+1}^{n}\left(-i \alpha^{\prime}\left|\vec{p}_{c}\right| \zeta_{i} \hat{p}_{c}\right)
$$


As a basis for the polarizations of the massless closed string it is natural to use 24 spacelike vectors orthogonal to the time direction $\hat{t}$ and to $\hat{p}_{c}$. The same 24 spacelike vectors together with $\hat{p}_{c}$ provide a basis for the polarizations of the massive open state. In this basis

$$
\epsilon D p_{c}=\bar{\epsilon} D p_{c}=\epsilon p_{o}=\bar{\epsilon} p_{o}=0,
$$

and the previous expression reduces to

$$
B_{g, S_{n}}=\beta \frac{(-1)^{n+1}}{\sqrt{n !}}\left(\frac{n-1}{2}\right)^{\frac{n}{2}} \epsilon_{\mu} \bar{\epsilon}_{\nu}\left[D^{\mu \nu} \zeta_{1} \hat{p}_{c} \zeta_{2} \hat{p}_{c}+2 n \eta^{\mu \alpha_{1}} \zeta_{\alpha_{1}} D^{\nu \alpha_{2}} \zeta_{\alpha_{2}}\right] \prod_{i=3}^{n}\left(\zeta_{i} \hat{p}_{c}\right)
$$

where we used that $\alpha^{\prime} \vec{p}_{c}{ }^{2}=n-1$. Let us analyze in detail the transition to the first massive level $(n=2)$

$$
B_{g, S_{2}}=-\beta \frac{1}{2 \sqrt{2}} \epsilon_{\mu} \bar{\epsilon}_{\nu}\left[D^{\mu \nu} \zeta_{\rho \sigma} \hat{p}_{c}^{\rho} \hat{p}_{c}^{\sigma}+4 \zeta^{\mu}{ }_{\alpha} D^{\alpha \nu}\right],
$$

where $\zeta_{\rho \sigma}$ is symmetric and traceless. We discuss separately the excitation of an open string with polarization transverse to $\hat{p}_{c}$ and parallel to $\hat{p}_{c}$

$$
B_{g, S_{2}, \text { tr }}=-\sqrt{2} \beta \epsilon_{\mu} D \bar{\epsilon}_{\nu} \zeta^{\mu \nu}, \quad B_{g, S_{2}, \text { lon }}=-\frac{\beta}{\sqrt{12}} \epsilon_{\mu} \bar{\epsilon}_{\nu} D^{\mu \nu},
$$

and analyze in turn the absorption of a dilaton $\varphi$, a graviton $G$ and a Kalb-Ramond field $B$. The dilaton can only excite longitudinally polarized states with amplitude ${ }^{15}$

$$
B_{g, S_{2}, \text { lon }}^{\varphi}=\frac{\beta}{\sqrt{72}}(12-p) .
$$

The graviton and the Kalb-Ramond field can only excite states with transverse polarization. The graviton can be absorbed when its indices are both parallel to the brane or both orthogonal to the brane and the collision axis

$$
B_{g, S_{2}, \mathrm{tr}}^{G_{\|,\|}}=-\sqrt{2} \beta, \quad B_{g, S_{2}, \mathrm{tr}}^{G_{\|, \perp}}=0, \quad B_{g, S_{2}, \mathrm{tr}}^{G_{\perp, \perp}}=\sqrt{2} \beta,
$$

while the Kalb-Ramond field can be absorbed when one of its indices is parallel to the brane and the other is orthogonal to the brane and the collision axis

$$
B_{g, S_{2}, \mathrm{tr}}^{B_{\|,\|}}=0, \quad B_{g, S_{2}, \mathrm{tr}}^{B_{\|, \perp}}=-\sqrt{2} \beta, \quad B_{g, S_{2}, \mathrm{tr}}^{B_{\perp, \perp}}=0 .
$$

If the massive polarization is longitudinal we find

$$
B_{g, S_{2}, l}=-\frac{\beta}{\sqrt{12}} \epsilon_{\mu} \bar{\epsilon}_{\nu} D^{\mu \nu} .
$$

We see that only the dilaton couples since all the other closed polarizations are traceless ${ }^{16}$

$$
B_{g, S_{2}, l}=\frac{\beta}{\sqrt{12}} \frac{24-2 p}{\sqrt{24}}=\frac{\beta}{\sqrt{72}}(12-p) .
$$

\footnotetext{
${ }^{15}$ Here $p$ is the dimension of the $\mathrm{D} p$-branes.

${ }^{16}$ Here $p$ is the dimension of the $\mathrm{D} p$-branes.
} 
The basis of physical polarizations that we used in the previous discussion coincides with the basis of light-cone polarizations for a massless state with $\vec{p}_{t}=0$. In this case the vertex gives

$$
V_{0}\left|g_{\epsilon}, \bar{g}_{\bar{\epsilon}}\right\rangle=\beta\left[N_{11}^{13} N_{11}^{23} \epsilon_{i} D \bar{\epsilon}_{j} a_{-1}^{i} a_{-1}^{j}+\frac{1}{2} \epsilon_{k} D \bar{\epsilon}_{k} N_{11}^{12} N_{11}^{33} a_{-1}^{i} a_{-1}^{i}\right]
$$

where

$$
N_{11}^{13}=-\frac{1+\rho}{2}=-1, \quad N_{11}^{23}=N_{11}^{12}=1, \quad N_{11}^{33}=-\frac{1-\rho^{2}}{8}=0,
$$

since $\rho=\frac{p}{E}=1$. Therefore

$$
V_{0}\left|g_{\epsilon}, \bar{g}_{\bar{\epsilon}}\right\rangle=-\beta \epsilon_{i} D \bar{\epsilon}_{j} a_{-1}^{i} a_{-1}^{j}
$$

Consider now the following basis of light-cone states for the first massive level:

$$
\left|\chi_{\mathrm{tr}}\right\rangle=\frac{\omega_{i j}}{\sqrt{2}} a_{-1}^{i} a_{-1}^{j}, \quad\left|\chi_{\mathrm{lon}}\right\rangle=\frac{1}{\sqrt{48}} a_{-1}^{i} a_{-1}^{i},
$$

with $\omega_{i j}$ a symmetric traceless tensor in 24 space directions. Then we find

$$
\begin{aligned}
\left\langle\chi_{\mathrm{tr}}\left|V_{0}\right| g_{\epsilon}, \bar{g}_{\bar{\epsilon}}\right\rangle & =-\sqrt{2} \beta \epsilon_{i} D \bar{\epsilon}_{j} \omega^{i j}, \\
\left\langle\chi_{\mathrm{lon}}\left|V_{0}\right| g_{\epsilon}, \bar{g}_{\bar{\epsilon}}\right\rangle & =-\frac{\beta}{\sqrt{12}} \epsilon_{i} \bar{\epsilon}_{j} D^{i j},
\end{aligned}
$$

in perfect agreement with the covariant amplitude.

Let us now consider $\vec{p}_{t} \neq 0$. In this case the basis of light-cone polarizations is different from the basis naturally associated to the states in the covariant calculation. The vertex gives

$$
\begin{aligned}
V_{\vec{p}_{t}}\left|g_{\epsilon}, \bar{g}_{\bar{\epsilon}}\right\rangle= & \beta\left[N_{11}^{13} N_{11}^{23} \epsilon_{i} D \bar{\epsilon}_{j} a_{-1}^{i} a_{-1}^{j}+\alpha_{3}^{2} N_{1}^{1} N_{11}^{23} N_{1}^{3} \frac{\alpha^{\prime}}{2}\left(\epsilon \vec{p}_{t}\right) D \bar{\epsilon}^{i} p_{t}^{j} a_{-1}^{i} a_{-1}^{j}\right. \\
& +\alpha_{3} N_{1}^{1} N_{12}^{23} \sqrt{\frac{\alpha^{\prime}}{2}}\left(\epsilon \vec{p}_{t}\right) D \bar{\epsilon}^{i} a_{-2}^{i}+\alpha_{3}^{2} N_{1}^{2} N_{11}^{13} N_{1}^{3} \frac{\alpha^{\prime}}{2}\left(D \bar{\epsilon} \vec{p}_{t}\right) \epsilon^{i} p_{t}^{j} a_{-1}^{i} a_{-1}^{j} \\
& +\alpha_{3} N_{1}^{2} N_{12}^{13} \sqrt{\frac{\alpha^{\prime}}{2}}\left(D \bar{\epsilon} \vec{p}_{t}\right) \epsilon^{i} a_{-2}^{i}+\frac{1}{2}\left(\epsilon \in \bar{\epsilon}_{k} N_{11}^{12}+\alpha_{3}^{2} N_{1}^{1} N_{1}^{2} \frac{\alpha^{\prime}}{2}\left(\epsilon \vec{p}_{t}\right)\left(D \bar{\epsilon} \vec{p}_{t}\right)\right) \\
& \left.\left(N_{11}^{33} a_{-1}^{i} a_{-1}^{i}+\frac{\alpha^{\prime}}{2} \alpha_{3}^{2}\left(N_{1}^{3}\right)^{2} p_{t}^{i} p_{t}^{j} a_{-1}^{i} a_{-1}^{j}+\sqrt{2 \alpha^{\prime}} \alpha_{3} N_{2}^{3} p_{t}^{i} a_{-2}^{i}\right)\right]
\end{aligned}
$$

where

$$
\begin{aligned}
& N_{11}^{13}=-\frac{1+\rho}{2}, \\
& N_{11}^{23}=N_{11}^{12}=1, \\
& N_{11}^{33}=-\frac{1-\rho^{2}}{8}, \\
& \alpha_{3} N_{1}^{1}=-\frac{2}{1+\rho}, \\
& \alpha_{3} N_{1}^{2}=-\frac{2}{1-\rho}, \\
& N_{12}^{13}=-\frac{1-\rho}{2}, \\
& N_{12}^{23}=\frac{1+\rho}{2}, \\
& \alpha_{3} N_{1}^{3}=1 \text {, } \\
& \alpha_{3} N_{2}^{3}=\frac{\rho}{2},
\end{aligned}
$$


and

$$
\rho=\frac{p}{E}=\sqrt{1-\alpha^{\prime} \vec{p}_{t}^{2}} .
$$

In the limit $\vec{p}_{t} \rightarrow 0$ we find

$$
V_{\vec{p}_{t}}\left|g_{\epsilon}, \bar{g}_{\bar{\epsilon}}\right\rangle \rightarrow-\beta \epsilon_{i}\left(D \bar{\epsilon}_{j}-2\left(D \bar{\epsilon} \hat{p}_{t}\right) \hat{p}_{t}^{j}\right) a_{-1}^{i} a_{-1}^{j}
$$

We see that the result of the limit depends on the direction of $\vec{p}_{t}$. It does not agree with eq. (A.33) and does not reproduce the covariant amplitudes. The correct limit is found if we give a small mass $\mu$ to the closed state setting

$$
\rho(\mu)=\frac{p}{E}=\sqrt{1-\alpha^{\prime}\left(\vec{p}_{t}^{2}+\mu^{2}\right)},
$$

and then send $\vec{p}_{t}$ to zero before removing the mass.

\section{B On the calculation of $\operatorname{Im} \mathcal{A}$ from the vertex}

In this appendix we describe in more detail how to derive the imaginary part of the disk starting from the closed-open vertex. We will discuss the absorption of the massive states $|L, \bar{L}\rangle$ and $|H, \bar{H}\rangle$, already analyzed in section 5. It is convenient to define

$$
\sigma_{\omega}(k) \equiv \frac{k^{-\omega \frac{k}{n}}}{\Gamma\left(1-\omega \frac{k}{n}\right)}, \quad \sigma_{\omega}(x) \equiv \frac{x^{-\omega x}}{\Gamma(1-\omega x)}, \quad \omega=N-1+\frac{\alpha^{\prime}}{4} \vec{p}_{t}^{2} .
$$

We also introduce the following compact notation for the integration over the $n$-simplex:

$$
\int d \mu_{n} \equiv \int_{0}^{1} d x_{1} \ldots \int_{0}^{1} d x_{n} \delta\left(\sum_{i=1}^{n} x_{i}-1\right)
$$

Let us begin with the evaluation of the first few terms in the series of the imaginary part of the elastic amplitude for the state $|L, \bar{L}\rangle$. The first contribution is

$$
\begin{aligned}
\operatorname{Im} \mathcal{A}_{L L} & \sim \frac{\pi}{4} \alpha^{\prime} \beta^{2} \zeta_{s^{\prime}} D \bar{\zeta}_{r^{\prime}} \epsilon_{s} D \bar{\epsilon}_{r} \sum_{\substack{u, k, l \\
v, k^{\prime}, l^{\prime}}} \delta_{u+k+l, n} N_{2 u}^{23} N_{2 v}^{23} N_{k l}^{33} N_{k^{\prime} l^{\prime}}^{33}\left\langle 0\left|a_{2}^{s^{\prime}} a_{v}^{r^{\prime}} a_{k^{\prime}}^{i} a_{l^{\prime}}^{i} a_{-k}^{j} a_{-l}^{j} a_{-u}^{r} a_{-2}^{s}\right| 0\right\rangle \\
& =\frac{\pi}{2} \alpha^{\prime} \beta^{2}(\zeta \epsilon) D \bar{\zeta}_{r^{\prime}} D \bar{\epsilon}_{r} \sum_{\substack{u, k, l \\
v, k^{\prime}, l^{\prime}}} \delta_{u+k+l, n} N_{2 u}^{23} N_{2 v}^{23} N_{k l}^{33} N_{k^{\prime} l^{\prime}}^{33}\left\langle 0\left|a_{v}^{r^{\prime}} a_{k^{\prime}}^{i} a_{l^{\prime}}^{i} a_{-k}^{j} a_{-l}^{j} a_{-u}^{r}\right| 0\right\rangle
\end{aligned}
$$

where we used the fact that only when the modes $a_{ \pm 2}$ are contracted among themselves we obtain a leading contribution. Performing the contractions between the modes in the vacuum expectation value in the second line of the previous equation and taking into account the symmetry of the coefficients $N_{k l}^{33}$ with respect to the exchange of the lower 
indices we find

$$
\begin{aligned}
\operatorname{Im} \mathcal{A}_{L L} & \sim \frac{\pi}{2} \alpha^{\prime} \beta^{2}(\zeta \epsilon)(\bar{\zeta} \bar{\epsilon}) \sum_{\substack{u, k, l \\
v, k^{\prime}, l^{\prime}}} \delta_{u+k+l, n} N_{2 u}^{23} N_{2 v}^{23} N_{k l}^{33} N_{k^{\prime} l^{\prime}}^{33}\left(48 k l u \delta_{u v} \delta_{k k^{\prime}} \delta_{l l^{\prime}}+4 u v l \delta_{u k} \delta_{v k^{\prime}} \delta_{l l^{\prime}}\right) \\
& =\frac{\pi}{2} \alpha^{\prime} \beta^{2}(\zeta \epsilon)(\bar{\zeta} \bar{\epsilon}) \sum_{u, k, l} \delta_{u+k+l, n} k l u\left[48\left(N_{2 u}^{23} N_{k l}^{33}\right)^{2}+4 N_{2 u}^{23} N_{2 k}^{23} N_{u l}^{33} N_{k l}^{33}\right] \\
& =\pi \alpha^{\prime} \beta^{2}(\zeta \epsilon)(\bar{\zeta} \bar{\epsilon}) \sum_{u, k, l} \delta_{u+k+l, n} k l u \frac{\left(\sigma_{1}(u) \sigma_{1}(k) \sigma_{1}(l)\right)^{2}}{\left(2-\frac{u}{n}\right)(k+l)}\left[\frac{96}{\left(2-\frac{u}{n}\right)(k+l)}+\frac{8}{\left(2-\frac{k}{n}\right)(u+l)}\right] .
\end{aligned}
$$

We now approximate the sums with integrals setting $u=n x_{1}, k=n x_{2}, l=n x_{3}$ and taking the large $n$ limit, as discussed in section 5

$$
\operatorname{Im} \mathcal{A}_{L L} \sim \pi \alpha^{\prime} \beta^{2} n(\zeta \epsilon)(\bar{\zeta} \bar{\epsilon})\left[96 I_{L, 1}+8 I_{L, 2}\right],
$$

where

$$
\begin{aligned}
& I_{L, 1}=\int d \mu_{3} x_{1} x_{2} x_{3}\left(\frac{\sigma_{1}\left(x_{1}\right) \sigma_{1}\left(x_{2}\right) \sigma_{1}\left(x_{3}\right)}{\left(2-x_{1}\right)\left(x_{2}+x_{3}\right)}\right)^{2} \sim 0.0082007 \\
& I_{L, 2}=\int d \mu_{3} \frac{x_{1} x_{2} x_{3}\left(\sigma_{1}\left(x_{1}\right) \sigma_{1}\left(x_{2}\right) \sigma_{1}\left(x_{3}\right)\right)^{2}}{\left(2-x_{1}\right)\left(2-x_{2}\right)\left(x_{1}+x_{3}\right)\left(x_{2}+x_{3}\right)} \sim 0.0066155 .
\end{aligned}
$$

The second contribution is proportional to

$$
\begin{aligned}
& \frac{1}{64} N_{2 u}^{23} N_{2 v}^{23} N_{k l}^{33} N_{g h}^{33} N_{k^{\prime} l^{\prime}}^{33} N_{g^{\prime} h^{\prime}}^{33}\left\langle 0\left|a_{2}^{s^{\prime}} a_{v}^{r^{\prime}} a_{k^{\prime}}^{i} a_{l^{\prime}}^{i} a_{g^{\prime}}^{j} a_{h^{\prime}}^{j} a_{-g}^{w} a_{-h}^{w} a_{-k}^{q} a_{-l}^{q} a_{-u}^{r} a_{-2}^{s}\right| 0\right\rangle \\
= & \frac{1}{32} N_{2 u}^{23} N_{2 v}^{23} N_{k l}^{33} N_{g h}^{33} N_{k^{\prime} l^{\prime}}^{33} N_{g^{\prime} h^{\prime}}^{33} \delta^{s s^{\prime}}\left[4 u g \delta^{r r^{\prime}} \delta^{i w} \delta_{u v} \delta_{g k^{\prime}}+16 u g \delta^{w r^{\prime}} \delta^{i r} \delta_{g v} \delta_{u k^{\prime}}\right] \\
& \times\left\langle 0\left|a_{l^{\prime}}^{i} a_{g^{\prime}}^{j} a_{h^{\prime}}^{j} a_{-h}^{w} a_{-k}^{q} a_{-l}^{q}\right| 0\right\rangle \\
= & \frac{1}{32} N_{2 u}^{23} N_{2 v}^{23} N_{k l}^{33} N_{g h}^{33} N_{k^{\prime} l^{\prime}}^{33} N_{g^{\prime} h^{\prime}}^{33} \delta^{r r^{\prime}} \delta^{s s^{\prime}} u g h k l\left(96 \delta_{u v} \delta_{g k^{\prime}}+16 \delta_{g v} \delta_{u k^{\prime}}\right) \\
& \times\left(48 \delta_{l^{\prime} h} \delta_{g^{\prime} k} \delta_{h^{\prime} l}+4 \delta_{g^{\prime} h} \delta_{h^{\prime} l} \delta_{k l^{\prime}}\right),
\end{aligned}
$$

where we left the sum understood. Setting $u=n x_{1}, g=n x_{2}, h=n x_{3}, k=n x_{4}, l=n x_{5}$ and taking the large $n$ limit we find

$$
n \delta^{r r^{\prime}} \delta^{s s^{\prime}}\left(576 I_{L, 3}+96 I_{L, 4}+48 I_{L, 5}+8 I_{L, 6}\right),
$$

where

$$
\begin{aligned}
I_{L, 3} & =\int d \mu_{5} x_{1} x_{2} x_{3} x_{4} x_{5}\left(\frac{\sigma_{1}\left(x_{1}\right) \sigma_{1}\left(x_{2}\right) \sigma_{1}\left(x_{3}\right) \sigma_{1}\left(x_{4}\right) \sigma_{1}\left(x_{5}\right)}{\left(2-x_{1}\right)\left(x_{2}+x_{3}\right)\left(x_{4}+x_{5}\right)}\right)^{2} \sim 0.0002179, \\
I_{L, 4} & =\int d \mu_{5} \frac{x_{1} x_{2} x_{3} x_{4} x_{5}\left(\sigma_{1}\left(x_{1}\right) \sigma_{1}\left(x_{2}\right) \sigma_{1}\left(x_{3}\right) \sigma_{1}\left(x_{4}\right) \sigma_{1}\left(x_{5}\right)\right)^{2}}{\left(2-x_{1}\right)\left(2-x_{2}\right)\left(x_{2}+x_{3}\right)\left(x_{4}+x_{5}\right)^{2}\left(x_{1}+x_{3}\right)} \sim 0.0001709, \\
I_{L, 5}= & \int d \mu_{5} \frac{x_{1} x_{2} x_{3} x_{4} x_{5}\left(\sigma_{1}\left(x_{1}\right) \sigma_{1}\left(x_{2}\right) \sigma_{1}\left(x_{3}\right) \sigma_{1}\left(x_{4}\right) \sigma_{1}\left(x_{5}\right)\right)^{2}}{\left(2-x_{1}\right)^{2}\left(x_{2}+x_{3}\right)\left(x_{2}+x_{4}\right)\left(x_{4}+x_{5}\right)\left(x_{3}+x_{5}\right)} \sim 0.0002087, \\
I_{L, 6}= & \int d \mu_{5} \frac{x_{1} x_{2} x_{3} x_{4} x_{5}\left(\sigma_{1}\left(x_{1}\right) \sigma_{1}\left(x_{2}\right) \sigma_{1}\left(x_{3}\right) \sigma_{1}\left(x_{4}\right) \sigma_{1}\left(x_{5}\right)\right)^{2}}{\left(2-x_{1}\right)\left(2-x_{2}\right)\left(x_{2}+x_{3}\right)\left(x_{4}+x_{5}\right)\left(x_{1}+x_{4}\right)\left(x_{3}+x_{5}\right)} \sim 0.0001667 .
\end{aligned}
$$


Adding the second contribution to the first we obtain

$$
\operatorname{Im} \mathcal{A}_{L L} \sim \pi \alpha^{\prime} \beta^{2} n(\zeta \epsilon)(\bar{\zeta} \bar{\epsilon})(0.841+0.153)=\pi \alpha^{\prime} \beta^{2} n(\zeta \epsilon)(\bar{\zeta} \bar{\epsilon}) 0.994 .
$$

We now give the details of the derivation of the higher order terms in the series expansion of the imaginary part of the disk for the state $|H, \bar{H}\rangle$. We start by rewriting eq. (5.69)

$$
\begin{aligned}
\operatorname{Im} \mathcal{A}_{H H} \sim & \pi \alpha^{\prime} \beta^{2}\left(\hat{\epsilon}_{i j} \hat{\zeta}_{i j}\right) \frac{1}{8}\langle 0|\left[n^{2} \hat{\bar{\zeta}}_{l l}+2 N_{1 u^{\prime}}^{23} N_{1 v^{\prime}}^{23}(D \hat{\bar{\zeta}} D)_{r^{\prime} s^{\prime}} a_{u^{\prime}}^{r^{\prime}} a_{v^{\prime}}^{s^{\prime}}\right] e^{Z_{o}^{\dagger}} \\
& \mathcal{P}_{n} e^{Z_{o}}\left[n^{2} \hat{\bar{\epsilon}}_{k k}+2 N_{1 u}^{23} N_{1 v}^{23}(D \hat{\bar{\epsilon}} D)_{r s} a_{-u}^{r} a_{-v}^{s}\right]|0\rangle .
\end{aligned}
$$

In addition to the term already evaluated in eq. (5.70) of section 5, we have the following terms with two summation variables:

$$
\pi \alpha^{\prime} \beta^{2}\left(\hat{\epsilon}_{i j} \hat{\zeta}_{i j}\right) \frac{1}{8} \sum_{k, l} \delta_{k+l, n}\left[4 k l N_{1 k}^{23} N_{1 l}^{23} N_{k l}^{33}+12 n^{2} k l\left(N_{k l}^{33}\right)^{2}\right] n^{2} \hat{\bar{\epsilon}}_{r r} \hat{\bar{\zeta}}_{s s}
$$

that in the continuum limit become

$$
\pi \alpha^{\prime} \beta^{2} n\left(\hat{\epsilon}_{i j} \hat{\zeta}_{i j}\right) \sum_{k, l} \delta_{k+l, n}\left[-\frac{1}{2} I_{H, 2}+\frac{3}{2} I_{H, 3}\right] \hat{\bar{\epsilon}}_{r r} \hat{\bar{\zeta}}_{s s}
$$

where

$$
\begin{aligned}
I_{H, 2} & =\int d \mu_{2} x_{1} x_{2} \frac{\left(\sigma_{1}\left(x_{1}\right) \sigma_{1}\left(x_{2}\right)\right)^{2}}{\left(1-x_{1}\right)\left(1-x_{2}\right)\left(x_{1}+x_{2}\right)} \sim 0.178692, \\
I_{H, 3} & =\int d \mu_{2} x_{1} x_{2} \frac{\left(\sigma_{1}\left(x_{1}\right) \sigma_{1}\left(x_{2}\right)\right)^{2}}{\left(x_{1}+x_{2}\right)^{2}} \sim 0.039863 .
\end{aligned}
$$

In eq. (B.13) we have obtained two terms that do not have the correct contraction for the polarizations as discussed in eq. (5.73). Therefore, let us go back to eq. (B.11) and include also higher order terms that come from the expansion of the two exponentials containing $Z_{o}$ and $Z_{0}^{\dagger}$. There are four terms with four summation variables that contribute to the imaginary part. The first one is

$$
\begin{aligned}
& \pi \alpha^{\prime} \beta^{2}\left(\hat{\epsilon}_{i j} \hat{\zeta}_{i j}\right)(D \hat{\bar{\zeta}} D)_{r^{\prime} s^{\prime}}(D \hat{\bar{\epsilon}} D)_{r s} \frac{1}{8} N_{1 u}^{23} N_{1 v}^{23} N_{k l}^{33} N_{1 u^{\prime}}^{23} N_{1 v^{\prime}}^{23} N_{k^{\prime} l^{\prime}}^{33}\left\langle 0\left|a_{u^{\prime}}^{r^{\prime}} a_{v^{\prime}}^{s^{\prime}} a_{k^{\prime}}^{i} a_{l^{\prime}}^{i} a_{-k}^{j} a_{-l}^{j} a_{-u}^{r} a_{-v}^{s}\right| 0\right\rangle \\
= & \pi \alpha^{\prime} \beta^{2} n\left(\hat{\epsilon}_{i j} \hat{\zeta}_{i j}\right)\left[\left(12 I_{H, 4}+2 I_{H, 5}\right)\left(\hat{\bar{\epsilon}}_{k l} \hat{\bar{\zeta}}_{k l}\right)+\frac{1}{2} I_{H, 6} \hat{\bar{\epsilon}}_{k k} \hat{\bar{\zeta}}_{l l}\right],
\end{aligned}
$$

where the three integrals correspond to three inequivalent contractions of the modes in the vacuum expectation value and are given by

$$
\begin{aligned}
I_{H, 4} & =\int d \mu_{4} x_{1} x_{2} x_{3} x_{4} \frac{\left(\sigma_{1}\left(x_{1}\right) \sigma_{1}\left(x_{2}\right) \sigma_{1}\left(x_{3}\right) \sigma_{1}\left(x_{4}\right)\right)^{2}}{\left(1-x_{1}\right)^{2}\left(1-x_{2}\right)^{2}\left(x_{3}+x_{4}\right)^{2}} \sim 0.012072, \\
I_{H, 5} & =\int d \mu_{4} x_{1} x_{2} x_{3} x_{4} \frac{\left(\sigma_{1}\left(x_{1}\right) \sigma_{1}\left(x_{2}\right) \sigma_{1}\left(x_{3}\right) \sigma_{1}\left(x_{4}\right)\right)^{2}}{\left(1-x_{1}\right)^{2}\left(1-x_{2}\right)\left(1-x_{3}\right)\left(x_{3}+x_{4}\right)\left(x_{2}+x_{4}\right)} \sim 0.007971, \\
I_{H, 6} & =\int d \mu_{4} x_{1} x_{2} x_{3} x_{4} \frac{\left(\sigma_{1}\left(x_{1}\right) \sigma_{1}\left(x_{2}\right) \sigma_{1}\left(x_{3}\right) \sigma_{1}\left(x_{4}\right)\right)^{2}}{\left(1-x_{1}\right)\left(1-x_{2}\right)\left(1-x_{3}\right)\left(1-x_{4}\right)\left(x_{1}+x_{2}\right)\left(x_{3}+x_{4}\right)} \sim 0.005733 .
\end{aligned}
$$


The second is

$$
\begin{aligned}
& \pi \alpha^{\prime} \beta^{2}\left(\hat{\epsilon}_{i j} \hat{\zeta}_{i j}\right) \hat{\bar{\zeta}}_{s s}(D \hat{\bar{\epsilon}} D)_{r^{\prime} s^{\prime}} \frac{n^{2}}{64} N_{1 u}^{23} N_{1 v}^{23} N_{k l}^{33} N_{k^{\prime} l^{\prime}}^{33} N_{g h}^{33}\left\langle 0\left|a_{k^{\prime}}^{i} a_{l^{\prime}}^{i} a_{g}^{j} a_{h}^{j} a_{-k}^{w} a_{-l}^{w} a_{-u}^{r^{\prime}} a_{-v}^{s^{\prime}}\right| 0\right\rangle \\
= & -\pi \alpha^{\prime} \beta^{2} n\left(\hat{\epsilon}_{i j} \hat{\zeta}_{i j}\right) \hat{\bar{\epsilon}}_{r r} \hat{\bar{\zeta}}_{s s}\left[3 I_{H, 7}+\frac{1}{4} I_{H, 8}\right]
\end{aligned}
$$

where the two integrals are

$$
\begin{aligned}
I_{H, 7} & =\int d \mu_{4} x_{1} x_{2} x_{3} x_{4} \frac{\left(\sigma_{1}\left(x_{1}\right) \sigma_{1}\left(x_{2}\right) \sigma_{1}\left(x_{3}\right) \sigma_{1}\left(x_{4}\right)\right)^{2}}{\left(1-x_{1}\right)\left(1-x_{2}\right)\left(x_{1}+x_{2}\right)\left(x_{3}+x_{4}\right)^{2}} \sim 0.008181 \\
I_{H, 8} & =\int d \mu_{4} x_{1} x_{2} x_{3} x_{4} \frac{\left(\sigma_{1}\left(x_{1}\right) \sigma_{1}\left(x_{2}\right) \sigma_{1}\left(x_{3}\right) \sigma_{1}\left(x_{4}\right)\right)^{2}}{\left(1-x_{1}\right)\left(1-x_{2}\right)\left(x_{3}+x_{4}\right)\left(x_{1}+x_{3}\right)\left(x_{2}+x_{4}\right)} \sim 0.007700
\end{aligned}
$$

The third gives a contribution identical to the second and the fourth is

$$
\begin{aligned}
& \pi \alpha^{\prime} \beta^{2}\left(\hat{\epsilon}_{i j} \hat{\zeta}_{i j}\right) \hat{\bar{\epsilon}}_{r r} \hat{\bar{\zeta}}_{s s} \frac{n^{4}}{512} N_{k l}^{33} N_{g h}^{33} N_{k^{\prime} l^{\prime}}^{33} N_{g^{\prime} h^{\prime}}^{33}\left\langle 0\left|a_{k^{\prime}}^{i} a_{l^{\prime}}^{i} a_{g^{\prime}}^{j} a_{h^{\prime}}^{j} a_{-k}^{w} a_{-l}^{w} a_{-g}^{t} a_{-h}^{t}\right| 0\right\rangle \\
= & \pi \alpha^{\prime} \beta^{2} n\left(\hat{\epsilon}_{i j} \hat{\zeta}_{i j}\right) \hat{\bar{\epsilon}}_{r r} \hat{\bar{\zeta}}_{s s}\left[9 I_{H, 9}+\frac{3}{4} I_{H, 10}\right],
\end{aligned}
$$

where the two integrals are

$$
\begin{aligned}
I_{H, 9} & =\int d \mu_{4} x_{1} x_{2} x_{3} x_{4} \frac{\left(\sigma_{1}\left(x_{1}\right) \sigma_{1}\left(x_{2}\right) \sigma_{1}\left(x_{3}\right) \sigma_{1}\left(x_{4}\right)\right)^{2}}{\left(x_{1}+x_{2}\right)^{2}\left(x_{3}+x_{4}\right)^{2}} \sim 0.007789 \\
I_{H, 10} & =\int d \mu_{4} x_{1} x_{2} x_{3} x_{4} \frac{\left(\sigma_{1}\left(x_{1}\right) \sigma_{1}\left(x_{2}\right) \sigma_{1}\left(x_{3}\right) \sigma_{1}\left(x_{4}\right)\right)^{2}}{\left(x_{1}+x_{2}\right)\left(x_{3}+x_{4}\right)\left(x_{1}+x_{3}\right)\left(x_{2}+x_{4}\right)} \sim 0.007538 .
\end{aligned}
$$

Collecting all the terms with two and four summation variables we then find

$$
\begin{aligned}
\operatorname{Im} \mathcal{A}_{H H} \sim & \pi \alpha^{\prime} \beta^{2} n\left(\hat{\epsilon}_{i j} \hat{\zeta}_{i j}\right)\left[\left(I_{H, 1}+12 I_{H, 4}+2 I_{H, 5}\right)\left(\hat{\bar{\epsilon}}_{k l} \hat{\bar{\zeta}}_{k l}\right)\right. \\
& \left.+\left(-\frac{1}{2} I_{H, 2}+\frac{3}{2} I_{H, 3}+\frac{1}{2} I_{H, 6}-6 I_{H, 7}-\frac{1}{2} I_{H, 8}+9 I_{H, 9}+\frac{3}{4} I_{H, 10}\right) \hat{\bar{\epsilon}}_{k k} \hat{\bar{\zeta}}_{l l}\right] \\
\sim & \pi \alpha^{\prime} \beta^{2} n\left(\hat{\epsilon}_{i j} \hat{\zeta}_{i j}\right)\left[0.993\left(\hat{\bar{\epsilon}}_{k l} \hat{\bar{\zeta}}_{k l}\right)-0.004 \hat{\bar{\epsilon}}_{k k} \hat{\bar{\zeta}}_{l l}\right] .
\end{aligned}
$$

The terms contributing to the first kind of contraction are all positive and seem to add to one. The sign of the terms contributing to the second kind of contraction alternates with the number of factors of $N^{33}$ and seem to add to zero. In fact the result of the sum is one order of magnitude smaller than the individual terms.

Open Access. This article is distributed under the terms of the Creative Commons Attribution License (CC-BY 4.0), which permits any use, distribution and reproduction in any medium, provided the original author(s) and source are credited. 


\section{References}

[1] J.M. Maldacena, The Large-N limit of superconformal field theories and supergravity, Int. J. Theor. Phys. 38 (1999) 1113 [Adv. Theor. Math. Phys. 2 (1998) 231] [hep-th/9711200] [INSPIRE].

[2] E. Witten, Anti-de Sitter space and holography, Adv. Theor. Math. Phys. 2 (1998) 253 [hep-th/9802150] [INSPIRE].

[3] S.S. Gubser, I.R. Klebanov and A.M. Polyakov, Gauge theory correlators from noncritical string theory, Phys. Lett. B 428 (1998) 105 [hep-th/9802109] [INSPIRE].

[4] G. D'Appollonio, P. Di Vecchia, R. Russo and G. Veneziano, High-energy string-brane scattering: Leading eikonal and beyond, JHEP 11 (2010) 100 [arXiv:1008.4773] [INSPIRE].

[5] G. D'Appollonio, P. Vecchia, R. Russo and G. Veneziano, Microscopic unitary description of tidal excitations in high-energy string-brane collisions, JHEP 11 (2013) 126 [arXiv:1310.1254] [INSPIRE].

[6] G. D'Appollonio, P. Di Vecchia, R. Russo and G. Veneziano, The leading eikonal operator in string-brane scattering at high energy, Springer Proc. Phys. 153 (2014) 145 [arXiv:1310.4478] [INSPIRE].

[7] G. D'Appollonio, P. Vecchia, R. Russo and G. Veneziano, Regge behavior saves String Theory from causality violations, JHEP 05 (2015) 144 [arXiv: 1502.01254] [INSPIRE].

[8] W. Black and C. Monni, High energy string-brane scattering for massive states, Nucl. Phys. B 859 (2012) 299 [arXiv:1107.4321] [INSPIRE].

[9] M. Bianchi and P. Teresi, Scattering higher spins off D-branes, JHEP 01 (2012) 161 [arXiv: 1108.1071] [INSPIRE].

[10] X.O. Camanho, J.D. Edelstein, J.M. Maldacena and A. Zhiboedov, Causality Constraints on Corrections to the Graviton Three-Point Coupling, JHEP 02 (2016) 020 [arXiv:1407.5597] [INSPIRE].

[11] D. Amati, M. Ciafaloni and G. Veneziano, Superstring Collisions at Planckian Energies, Phys. Lett. B 197 (1987) 81 [INSPIRE].

[12] D. Amati, M. Ciafaloni and G. Veneziano, Classical and Quantum Gravity Effects from Planckian Energy Superstring Collisions, Int. J. Mod. Phys. A 3 (1988) 1615 [InSPIRE].

[13] G. Veneziano, String-theoretic unitary S-matrix at the threshold of black-hole production, JHEP 11 (2004) 001 [hep-th/0410166] [INSPIRE].

[14] G. D'Appollonio, A semiclassical picture of the absorption of closed strings by D-branes, to appear.

[15] E. Cremmer and J. Scherk, Factorization of the Pomeron sector and currents in the dual resonance model, Nucl. Phys. B 50 (1972) 222 [inSPIRE].

[16] L. Clavelli and J.A. Shapiro, Pomeron factorization in general dual models, Nucl. Phys. B 57 (1973) 490 [INSPIRE].

[17] M.B. Green, J.H. Schwarz and L. Brink, Superfield Theory of Type II Superstrings, Nucl. Phys. B 219 (1983) 437 [INSPIRE].

[18] J.A. Shapiro and C.B. Thorn, BRST Invariant Transitions Between Closed and Open Strings, Phys. Rev. D 36 (1987) 432 [InSPIRE]. 
[19] M.B. Green and P. Wai, The Insertion of boundaries in world sheets, Nucl. Phys. B 431 (1994) 131 [INSPIRE].

[20] G. D'Appollonio et al., in preparation.

[21] M. Ademollo, A. D’Adda, R. D'Auria, E. Napolitano, P. Di Vecchia, F. Gliozzi et al., Unified Dual Model for Interacting Open and Closed Strings, Nucl. Phys. B 77 (1974) 189 [INSPIRE].

[22] I.R. Klebanov and L. Thorlacius, The Size of p-branes, Phys. Lett. B 371 (1996) 51 [hep-th/9510200] [INSPIRE].

[23] M.R. Garousi and R.C. Myers, Superstring scattering from D-branes, Nucl. Phys. B 475 (1996) 193 [hep-th/9603194] [INSPIRE].

[24] A. Hashimoto and I.R. Klebanov, Scattering of strings from D-branes, Nucl. Phys. Proc. Suppl. 55B (1997) 118 [hep-th/9611214] [InSPIRE].

[25] H. Kawai, D.C. Lewellen and S.H. Tye, A Relation Between Tree Amplitudes of Closed and Open Strings, Nucl. Phys. B 269 (1986) 1 [InSPIRE].

[26] S. Stieberger, Open \& Closed vs. Pure Open String Disk Amplitudes arXiv:0907.2211 [INSPIRE].

[27] S. Stieberger and T.R. Taylor, Closed String Amplitudes as Single-Valued Open String Amplitudes, Nucl. Phys. B 881 (2014) 269 [arXiv:1401.1218] [INSPIRE].

[28] M. Ademollo, A. Bellini and M. Ciafaloni, Superstring Regge Amplitudes and Emission Vertices, Phys. Lett. B 223 (1989) 318 [InSPIRE].

[29] M. Ademollo, A. Bellini and M. Ciafaloni, Superstring Regge Amplitudes and Graviton Radiation at Planckian Energies, Nucl. Phys. B 338 (1990) 114 [InSPIRE].

[30] R.C. Brower, J. Polchinski, M.J. Strassler and C.-I. Tan, The Pomeron and gauge/string duality, JHEP 12 (2007) 005 [hep-th/0603115] [INSPIRE].

[31] M. Ademollo, E. Del Giudice, P. Di Vecchia and S. Fubini, Couplings of three excited particles in the dual-resonance model, Nuovo Cim. A 19 (1974) 181 [InSPIRE]. 